INFERENTIAL PROCEDURES FOR MULTIFACETED COEFFICIENTS OF GENERALIZABILITY

by

MARSHA IYNN SCHROEDER

M.A., The University of British Columbia, 1982

A THESIS SUBMITTED IN PARTIAL FULFILMENT OF THE REQUIREMENTS FOR THE DEGREE OF DOCTOR OF PHILOSOPHY

in

THE FACULTY OF GRADUATE STUDIES

Department of Psychology

We accept this thesis as conforming

to the required standard

THE UNIVERSITY OF BRITISH COLUMBIA

September 1986

- Marsha Lynn Schroeder, 1986 
In presenting this thesis in partial fulfilment of the requirements for an advanced degree at the University of British Columbia, I agree that the Library shall make it freely available for reference and study. I further agree that permission for extensive copying of this thesis for scholarly purposes may be granted by the head of my department or by his or her representatives. It is understood that copying or publication of this thesis for financial gain shall not be allowed without my written permission.

Department of PSYCHOLOGY

The University of British Columbia 1956 Main Mall Vancouver, Canada V6T 193

Date SEPT. 29, 1986 


\title{
INFERENTIAL PROCEDURES FOR MULTIFACETED COEFFICIENTS OF GENERALIZABILITY
}

\begin{abstract}
Generalizability theory was developed by Cronbach as an alternative to classical test score reliability theory. Generalizability uses an experimental design approach to rellability that permits the systematic evaluation of several sources of error simultaneously. The coefficient of generalizability (CG) is a single number summarizing the dependability of the measurement process.

In the present study a normalizing transformation was first applied to a function of the CG. The delta method was applied to the transformed CGs for four different two-facet experimental design models to develop asymptotic variance expressions for the CGs.

The accuracy of the variance expressions was tested via Monte Carlo simulations. In these simulations the Type I error control was investigated. The majority of the simulations were conducted using a two-facet fully random experimental design, corresponding to a three-way random effects analysis of variance. A total of 81 combinations of sample size, facet conditions, and population CG values were investigated. The results suggested that the procedure generally was precise in its control of Type I error. The results were- somewhat less precise when only two facet conditions were sampled.

Five other side studies were conducted. Three of these used other two-facet models: a design with one fixed facet, a design with a finite facet, and a design with a nested facet. The results of these studies were similar to those found in the larger study; generally good Type I exror control was realized.

An additional study looked at the performance of the variance expression in the presence of negative variance component estimates. Results in this section of the study suggested that such negative component estimates did not adversely affect Type I error control. The final study investigated the performance of the variance expression with dichotomous data. The results indicated that Type I error control was not as precise with two facet conditions as it was with five or efght conditions. In these latter cases good error control was realized.
\end{abstract}


Table of Contents

Page

Abstract ii

List of Tables. vi

List of Figures ix

Acknowledgements $\quad x$

Chapter 1 - Introduction 1

The Object of Measurement 3

Universes $3 \ldots \ldots$

Components of Variance 4

$G$ and $D$ Studies 6

Two Kinds of Decisions; Two Kinds of Error Variance 7

The Coefficient of Generalizability 9

Estimating the CG 12

Generalizability Research and Application 13

$\begin{array}{ll}\text { Purpose of the Present Study } & 18\end{array}$

Chapter 2 - Mathematical Development 20

The Approximate Distribution of the CG 20

The Normalization of a Function of the CG 25

The Derivation of an

Asymptotic Variance Expression for the CG 26

Variance Expressions

for CGs Arising from Other Experimental Designs 29 
Chapter 3 - Method 36

Data Generation 36

Overview of the Study

for Design VII with Both Facets Random 38

Three Other Models 39

A Special Condition: Zero Variance Components 41

A Special Condition: Dichotomous Data 43

Testing the Adequacy of the Variance Expression 44

Chapter 4 - Results 49

$\begin{array}{ll}\text { Design VII with Both Facets Random } & 49\end{array}$

Design VII with Fixed Item Facet 57

Design VII with Finite Random Rater Facet 57

Design V-B with Both Facets Random 60

The Treatment of Negative Variance Components 61

Design VII with Dichotomous Data 62

Chapter 5 - Conclusions and

a Worked Example of the Procedures 67

Some Observations about the Empirical Results 67

Implications of the Study

for the Use of Generalizability Theory $\quad 70$

Limitations of the Present Study 72

Suggestions for Future Research 73

A Worked Example Using the Present Procedure 75

References $\quad 77$

$\begin{array}{ll}\text { Appendix A } & 84\end{array}$ 
List of Tables

Page

Table 1 Expected Mean Squares for Design VII with both Facets Random

Table 2 Analysis of Variance Results and Estimated

Variance Components for Psychopathy Data

Table 3 . Summary of Designs and Conditions for the

Empirical Investigation

Table 4 Proportion of the Standardized Estimates

Falling below Selected Percentile points of the Unit Normal Distribution and Actual

Proportion of Type I Errors for $\rho^{2}=.50$

(Design VII, both Facets Random)

Table 5 Proportion of the Standardized Estimates Falling below Selected Percentile points of the Unit Normal Distribution and Actual Proportion of Type I Errors for $\rho^{2}=.70$ (Design VII, both Facets Random)

Table 6 Proportion of the Standardized Estimates Falling below Selected Percentile Points of the Unit Normal Distribution and Actual Proportion of Type I Errors for $\rho^{2}=.90$ (Design VII, both Facets Random) 
Table 7 Mean Values and Overall Chi-square Values by Parameter Values and Facet Conditions (Design VII, both Facets Random) for

Selected Percentile Points of the Unit Normal Distribution and Three Levels of Type I Error

Table 8 Proportion of the Standardized Estimates Falling below Selected percentile points of the Unit Normal Distribution and Actual Proportion of Type I Errors (Design VII, Item Facet Fixed)

Table 9 Proportion of the Standardized Estimates Falling below Selected Percentile Points of the Unit Normal Distribution and Actual Proportion of Type I Errors (Design VII, Rater Facet Finite)

Table 10 Proportion of the Standardized Estimates Falling below Selected Percentile points of the Unit Normal Distribution and Actual Proportion of Type I Errors (Design V-B, both Facets Random) 
Table 11 Proportion of the Standardized Estimates

Falling below Selected Percentile Points of

the Unit Normal Distribution and Actual

Proportion of Type I Errors for Two

Treatments of Negative Variance Component

Estimates (Design VII, Both Facets Random)

Table 12 Proportion of the Standardized Estimates

Falling below Selected Percentile Points of

the Unit Normal Distribution and Actual

Proportion of Type I Errors for Dichotomized

Data and Comparable Conditions with

Continuous Data (Design VII, both Facets

Random) 
List of Figures

Page

Figure 1 Layout of Data for Design VII Analysis of Variance 


\section{Acknowledgements}

I would like to thank my thesis committee for their support and encouragement throughout the course of this project. I would like to thank Demetri Papageorgis for consenting to serve on my committee. I also wish to express my appreciation to Jim steiger for his many helpful comments and suggestions. I am also indebted to Todd Rogers for introducing me to Generalizability theory; I also wish to thank Todd for his careful reading of and thoughtful comments on previous drafts of this thesis. I especially would like to thank Ralph Hakstian for chairing my committee. His patient assistance and encouragement were instrumental to the completion of this thesis. Finally, I would Iike to thank Klaus Schroeder for his assistance with text preparation; I would also like to thank klaus for his considerable support throughout this project. 
Chapter 1

Introduction

Generalizability theory was proposed by Cronbach (Cronbach, Gleser, Nanda, \& Rajaratnam, 1972; Cronbach, Rajaratnam, \& Gleser, 1963; Gleser, Cronbach, \& Rajaratnam, 1965; Rajaratnam, Cronbach, \& Gleser, 1965) as a liberalization of classical test score reliability theory. Classical test score theory is based on the assumption of parallel measurements; generalizability theory does not rely on this restrictive assumption. Measurement error within the classical model is regarded as an amorphous quantity; in generalizability theory measurement error is examined systematically.

The view of measurement error as an amorphous quantity has been questioned by a number of writers (e.g., Cronbach, 1947, 1970; Gulliksen, 1936). Errors arising from different sources may not be equivalent; for example, error arising from repeated testing with the same form of an instrument likely differs from error arising when parallel forms of the instrument are used. Generalizability theory permits the simultaneous evaluation of several sources of error by using an experimental design approach to measurement.

The next sections of this thesis will introduce generalizability theory in some detail. Following this presentation, inferential procedures pertaining to generalizability theory will be developed. 
To introduce the notion of an experimental design approach to measurement, consider the following example taken from a study by Schroeder, Schroeder, and Hare (1983). In this study the generalizability (reliability) of a checklist used to measure psychopathy in prison inmates was assessed. The checklist is composed of 22 items tapping aspects of psychopathy. It is typically used by two or more trained raters who judge the applicability of each item to a particular inmate. In each of five years a number of inmates were evaluated on the 22 items by two raters. In what follows we will be concerned with the data for a single year. If reliability were assessed using the classical test score model, a number of different indexes of reliability would be calculated. For example an index of internal consistency for each rater, a coefficient of interrater agreement, the significance of differences between raters on the means and variances for total test scores (i.e., the sum of the ratings given over the 22 items), and individual item statistics could be evaluated. Using generalizability theory, we would conceptualize these data as a three-way random effects analysis of variance (ANOVA) and conduct the corresponding Persons (i.e., inmates) X Items $X$ Raters fully-crossed ANOVA. The resulting mean squares are used to calculate the seven separate variance component estimates. These variance estimates are the key elements of generalizability theory; they guide the researcher in the design of sound data collection procedures. They are also 
used to compute the value of the coefficient of generalizability (CG), a single number summarizing the reliability or dependability of the measurement process.

At this point some generalizability theory concepts and terminology are presented to help clarify the material that follows.

\section{The Object of Measurement}

The object of measurement in generalizability theory is the element of the study about which one wishes to make judgments. In the Schroeder et al. (1983) study the objects of measurement were inmates. Throughout this thesis the objects of measurement will be referred to as persons. The objects of measurement in a generalizability study, however, could be any population of organisms or objects, to be evaluated. For example, the objects of measurement could be paintings or animals.

\section{Universes}

The emphasis in both classical test score theory and generalizability theory is placed on generalizing beyond the conditions used in the study to a larger set of conditions.

Any observation is made under a set of conditions. Facets are composed of conditions (the term facet is analogous to the ANOVA term factor; the term condition to (evel). An observation can be classified according to the 
conditions of the facets under which it was made. The universe of admissible observations consists of all conditions of a facet that theoretically could be included in a study. The universe of generalization represents all conditions of the facet over which one wishes to generalize. These two terms may be synonymous; however, in some situations the universe of generalization is a subset of the universe of admissible observations.

In a generalizability study, facets over which the researcher would like to generalize are identified. Two or more conditions of each facet are sampled for inclusion in the study. Facets can be fixed, random, or finite effects. When a facet is fixed, all conditions of the universe of admissible observations are included in the study. When a facet is random, a number of conditions are sampled randomly from the theoretically infinite universe of admissible observations. When a finite facet is included in a study, a number of conditions are sampled at random from a finite universe of admissible observations.

Related to the concept of universes is the concept of universe score. The universe score is like the classical test score notion of true score.

Components of Variance

The variance components are the building blocks of generalizability theory. Consider, for example, a matrix containing data (persons' scores on items) obtained from the 
administration of a test. This design is described in generalizability theory as a one-facet (Items) design. The object of measurement, persons, is not considered a facet even though, as shown next, it is included as a factor in the corresponding analysis of variance. In a basic one-facet design where each person rates him/herself for is scored, on the same set of items and where Items is considered as a random facet) the total observed score variance can be expressed as the sum of three variance components:

$$
\sigma^{2}\left(\mathrm{x}_{\mathrm{p} i}\right)=\sigma_{\mathrm{p}}^{2}+\sigma_{i}^{2}+\sigma_{\mathrm{pi}, \epsilon}^{2}
$$

In this model $\sigma^{2}$, the universe score variance, is variance due to differences among respondents, the objects of measurement; this variance is like true score variance in classical test score theory. The variance associated with items, $\sigma_{i}^{2}$, reflects differences among the items; item heterogeneity can indicate differing levels of item difficulty or social desirability. The term $\sigma_{p i, \epsilon}^{2}$ is the residual variance which is composed of the two-way Person by Item interaction plus error due to facets other than those explicitly considered facets and random fluctuation.

The Schroeder et al. (1983) design is an items by raters fully-crossed two-facet design. For this design the 
total observed score variance can be expressed as:

$$
\sigma^{2}\left(x_{p i r}\right)=\sigma_{p}^{2}+\sigma_{i}^{2}+\sigma_{r}^{2}+\sigma_{p i}^{2}+\sigma_{p r}^{2}
$$

$$
+\sigma_{\text {ir }}^{2}+\sigma_{\text {pir, } \epsilon}^{2}
$$

The variance components in equations (1) and (2) are estimated using from statistical theory the expressions for the ANOVA expected mean squares. The obtained mean squares are unbiased estimates of the expected mean squares. By substituting the values for the mean squares in the equations for the expected mean squares, estimates of the variance components can be calculated. For example, the estimate for $\sigma_{p}^{2}$ is given by

$$
\hat{\sigma}_{p}^{2}=\left(M S_{p}-M S_{p i}-M S_{p r}+M S_{p i r, \epsilon}\right)
$$

for the two-facet random effects model. The form of the expressions for the expected mean squares depends on whether the facets are fixed, random, or finite. Millman and Glass (1967) provided rules for writing these expressions (also see Cronbach et al., 1972, Ch. 2).

\section{$\underline{G \text { and } D \text { Studies }}$}

Cronbach et al. (1972) distinguished between two types of study. The first, the generalizability or G study, is conducted to obtain estimates of the variance components to be used to plan the second, the decision or $D$ study. The purpose of the D study is to make decisions about the object 
of measurement. The $D$ study is planned using information provided in the G study variance component estimates. Cronbach et al. viewed the $G$ and $D$ studies as being ideally distinct. They believed that large sample $G$ studies should be conducted with the resultant variance components being used to design other studies. In practice, however, the same data usually are used for both the $G$ and $D$ studies.

\section{Two Kinds of Decision; Two Kinds of Error Variance}

In the $D$ study one of two kinds of decision is made. An absolute decision is made when an individual's universe score estimate is compared to a criterion or cutting score. In the Schroeder et al. (1983) study if an inmate were classified as a psychopath when he received a mean rating of at least 2.8 out of a maximum of 3 , an absolute decision would have been made. The decision is absolute in the sense that no direct comparisons among the inmates tested were made. A relative decision is made when the individuals are rank ordered according to their estimated universe score. The relative standing of an individual is then used to make a decision about her or his future treatment. In the Schroeder et al. Study if the highest scoring $20 \%$ of the sample had been designated psychopaths, a relative decision would have been made.

Just as the two kinds of decision differ, so too do the estimates of error variance associated with the estimates of universe score upon which the decisions are based. In the 
case of absolute decisions, error variance arises from all sources of variance except the source due to the object of measurement. Cronbach et al. (1972) labelled this error variance $\sigma^{2}(\Delta)$. In the case of relative decisions, labelled by cronbach et al. as $\sigma^{2}(\delta)$, some sources of variance other than that due to the object of measurement do not enter into the expression for the error variance. Variance due to Items in the Schroeder et al. (1983) study, for example, reflects item heterogeneity. This source of variance is considered constant across all inmates (possible item-inmate interaction is accounted for in the item-person interaction variance component). When all facets of the design are random, $\sigma^{2}(\delta)$ incorporates all variance components involving interaction with the object of measurement. In contrast, in the case of a design incorporating a fixed facet, interaction of the object of measurement with a fixed facet is not considered to be error variance. Variance due to interactions between the object of measurement and a finite facet is considered error.

For both kinds of error variance, each constituent variance component is divided by the total number of conditions within the object of measurement upon which the variance is based. This procedure reflects the fact that the universe score estimate is the average value of the observations within the object of measurement. This procedure is equivalent to using the Spearman-Brown correction for test length (Cronbach et al., 1972, p. 82). 
In the Schroeder et al. study each inmate's universe score estimate is the average value taken over 44 observations ( 22 items for each of two raters).

In the present research, emphasis is placed on the error variance $\sigma^{2}(\delta)$. This conceptualization of error variance is closer to the classical test score notion of error variance than is the $\sigma^{2}(\Delta)$ error variance. This error is also of relevance in the study of individual differences where comparisons among persons are made. The Coefficient of Generalizability (CG)

The coefficient of generalizability, CG, is an intraclass correlation coefficient summarizing the adequacy of the measurement procedure in the case of relative decisions. The intraclass correlation coefficient is the correlation between exchangeable measurements obtained on the same object (Cronbach et al., 1972, p. 17; Shrout \& Fleiss, 1979). A negatively biased but consistent statistic (Lahey, Downey, \& Saal, 1983), the maximum value of the intraclass correlation coefficient is one; the theoretical lower limit is zero. Although the standard error of measurement is often used to summarize the adequacy of measurement, the CG presents the advantage of having a standardized metric.

As an example, consider again a simple one-facet design in which a number of persons are tested or evaluated on a number of items measuring some attribute. Further, suppose 
that the $n_{i}$ items were sampled at random from a theoretically infinitely large item pool for each individual (i.e., each individual receives a different set of items). These data would be analysed as a one-way random effects ANOVA with Persons as the Between factor in the design. The intraclass correlation coefficient for this design is the ratio of the between class variance (here person variance) to the total variance (Haggard, 1958, p. 4). The expression for the population value is:

$$
R=\sigma_{p}^{2} /\left[\sigma_{p}^{2}+\sigma_{i: p}^{2}\right]
$$

where $\sigma_{p}^{2}$ is the variance due to Persons, and $\sigma_{i: p}^{2}$ is the variance due to Items nested within Persons.

In Haggard's treatment of the intraclass correlation coefficient attention is focused on the individual item or observation as the unit of analysis (his treatment of the topic encompasses more than psychometric applications). Cronbach et al.'s treatment focuses on scores averaged over the conditions of facets; here the intraclass correlation coefficient (i.e., the CG) is

$$
\rho^{2}=\sigma_{p}^{2} /\left[\sigma_{p}^{2}+\sigma_{i: p}^{2} / n_{i}\right]
$$

Thus, Haggard's coefficient, $R$, represents the average reliability of a single item while cronbach et al.'s coefficient, $\rho^{2}$, represents the reliability of the aggregated items. 
Cronbach et al. (1972, p. 17) defined the CG as the ratio of universe score variance to expected observed score variance. This value is approximately equal to the squared correlation between the observed score and the universe score (Cronbach et al., p. 82 ), and as such is a coefficient of determination. The CG can be expressed more generally as

$$
\rho^{2}=\sigma^{2}(\tau) /\left[\sigma^{2}(\tau)+\sigma^{2}(\delta)\right]
$$

where $\sigma^{2}(\tau)$ is universe score variance and $\sigma^{2}(\delta)$ is, as defined previously, error variance associated with relative decisions.

The $C G$ is comparable to classical test score reliability coefficients (Cronbach et al., 1972, p. 84) and is interpreted the same way. The magnitude of the coefficient, $\rho^{2}$, indicates how reliably the object of measurement (e.g., the $n_{p}$ persons) can be rank ordered with the facets and the numbers of facet conditions used in the design. For example, in the Schroeder et al. (1983) study, the obtained value of $\hat{\rho}^{2}=.86$ (for a sample of 71 inmates) indicates that inmates can be reliably ranked using the average rating assigned by two raters on the 22 item checklist. Following Nunnally's (1978, pp. 245-246) guidelines for reliability, a coefficient of at least .7 is acceptable for research purposes where group averages are the locus of interest. In applied settings, where decisions will be made about individuals, reliability (generalizability) should be as high as possible. 
Estimating the CG

In practice, the $C G$ is estimated from the variance components calculated in the $G$ study. The calculated CG gives the researcher an estimate of the generalizability of a proposed D-study data collection procedure. From the variance component estimates the researcher can evaluate the relative adequacy of a variety of designs for data collection prior to actually conducting the study. This is accomplished by varying the number of facet conditions used in the computation of the CG. The magnitude of the coefficients that will constitute $\sigma^{2}(\delta)$ guides the researcher in determining whether more or fewer conditions of a particular facet should be sampled. If the estimate of a variance component contained within $\sigma^{2}(\delta)$ is small, it will contribute little to error variance. In this case only a few levels of the facet need to be sampled. In contrast, if a particular component estimate accounts for a large proportion of the error variance, its impact can be diminished by planning a $D$ study with a large number of conditions or seeking ways to diminish the value of the variance itself.

For example, in the Schroeder et al. (1983) example, the general form of the estimated CG is expressed as:

$$
\hat{\rho}^{2}=\hat{\sigma}_{\mathrm{p}}^{2} /\left[\hat{\sigma}_{\mathrm{p}}^{2}+\hat{\sigma}_{\mathrm{pi}}^{2} / \mathrm{n}_{\mathrm{i}}+\hat{\sigma}_{\mathrm{pr}}^{2} / \mathrm{n}_{\mathrm{r}}+\hat{\sigma}_{\mathrm{pir}, \epsilon}^{2} / \mathrm{n}_{\mathrm{i}} \mathrm{n}_{\mathrm{r}}\right]
$$


If it is found that the component $\hat{o}_{\mathrm{pr}}^{2}$, corresponding to the person by rater interaction, is large, the impact of this component can be minimized by employing a large number of raters in the $D$ study. If this approach is not practical, then steps should be taken to improve the quality of the data by improving the training procedures, thereby decreasing the value of $\sigma_{\mathrm{pr}}^{2}$.

It should be noted here that the choice of facets studied depends upon the purpose of the study. The Schroeder et al. (1983) example focused on the equivalence of raters. In other testing situations the stability of measurement over time may be of interest. In such a case an Occasions facet would be included in the generalizability study. Also it should be noted that several facets can be included in the same design. Further, as suggested in an earlier example, some facets can be nested, either because the study was so designed or because facets are naturally nested within some other facet--such as classes nested within Schools.

Generalizability Theory Research and Application

The use of generalizability theory has been advocated by a number of authors within the field of psychology (e.g., Jackson \& Paunonen, 1980; Mitchell, 1979; Wiggins, 1973). These authors stressed the advantages of a multifaceted approach to reliability estimation over the traditional classical test score approach. 
Pedagogical articles demonstrating the application of the generalizability approach have appeared in the social science literature. Cardinet, Tourneur, and Allal (1976, 1981) and Rentz (1980) presented detailed demonstrations of the application of the technique to educational measurement. Brennan and Kane (1979) presented a summary of the essential concepts and features of generalizability theory along with an example of a two-facet design application.

Applications of generalizability theory in research settings appear in the social science literature. In the psychological literature a number of authors (e.g., Chalmers \& Knight, 1985; Farrell, Mariotto, Conger, Curran, \& Wallander, 1979; Hansen, Tisdelle, \& O'Dell 1985; Wallander, Conger, \& Conger, 1985) have used a generalizability approach to estimate reliability in multifaceted instrument development studies. Generalizability theory applications also are found in the educational (e.g., Gillmore, Kane, \& Naccarato, 1978; Kane \& Brennan, 1977; Nussbaum, 1984) and organizational behaviour (e.g., Cain \& Green, 1983; Doverspike \& Barrett, 1984; Fraser, Cronshaw, \& Alexander, 1984) literature.

Although little research has focused on the statistical properties of the generalizability technique, some work has been directed toward examining the sampling properties of the variance component estimates. Shavelson and Webb (1981, p. 138) referred to these components as the "Achilles heel" 
of generalizability theory because of their sampling properties. Smith (1978) empirically investigated the sampling errors of variance component estimates under two design models. His results indicated that variance component estimates based on small numbers of facet conditions are unstable. G studies involving small numbers of conditions thus do not provide good estimates from which to predict the adequacy of subsequent $D$ studies. Leone and Nelson (1966) performed a similar study using hierarchical (completely nested) designs. Their results also indicated a high degree of sampling variability in the estimates that were based on linear combinations of mean squares.

Very little research has been directed toward investigating the sampling error of the CG. One reason for this neglect likely is that cronbach et al.(1972) placed relatively little emphasis on the coefficient; they stressed the greater importance of the variance components. They suggested that large scale $G$ studies should be conducted to provide stable variance component estimates. Such studies would involve sampling large numbers of facet conditions. The variance components calculated from such studies would then be used to estimate the generalizability of the proposed design. Because component estimates based on large numbers of facet conditions likely are stable, the CGs calculated with these components likely would also be stable. 
However, in many testing situations sufficient resources are not available to conduct a preliminary G study. Nor, generally, are suitable published G study results available. Even if $G$ study results were available, researchers would be advised to exercise caution in using other researchers' variance component estimates. Such estimates may be unstable (cf. Smith, 1978). Further, hidden or unmeasured facets, such as geographic location or occasion of testing, could have an impact on the $D$ study.

Most published research using generalizability theory is based on a single data collection that serves as both the $D$ and $G$ study. Researchers tend to place greater emphasis on the CG than they do on the variance component estimates. This probably reflects the long-standing psychometric tradition of reporting reliability and validity coefficients in instrument development studies. In addition, coefficients summarizing the adequacy of measurement often are needed in studies in which a new instrument has been developed for experimental purposes.

Researchers (e.g., Doverspike, Carlisi, Barrett, \& Alexander, 1983; Kane, Gillmore, \& Crooks, 1976) often report the estimated CG for the number of conditions used in the study as well as estimates for other numbers of conditions. Some authors have tended to treat these estimated CGs as if they were parameter values, rather than estimates. Doverspike et al., for example, stated that 
"...reliability dropped only slightly when the number of raters was reduced from 10 to $1 "$ (p. 481). However, no attention was given to the presence of sampling error in the estimates considered. To clarify such conclusions, it is important to report a confidence interval or the standard error for each coefficient, thereby indicating a measure of uncertainty due to sampling. A high value of an estimated CG does not necessarily guarantee that the measurement procedure is adequately reliable or that a similarly favourable coefficient would be found upon replication of the D study. But, by reporting say the $90 \%$ confidence interval, researchers would be provided with the likely range of the population parameter.

Some research has been directed toward examining the inferential properties of CGs computed for single facet studies. These studies have not dealt with generalizability theory, per se; they have investigated the properties of intraclass correlation coefficients and coefficient alpha--both of which are equivalent to CGs. Feldt (1965, 1969) presented inferential techniques for constructing single-sample confidence intervals and for making two independent sample comparisons for coefficient alpha (a one-facet crossed design in generalizability terminology). This work was extended by Hakstian and Whalen (1976) who developed a $k$ independent sample significance test for alpha coefficients. Fleiss and Shrout (1978; Shrout \& Fleiss, 1979) developed approximate confidence intervals for six 
intraclass correlation coefficients. More recently, Hakstian and Lind (1982) derived approximate variance and covariance expressions for coefficient alpha. These authors used the expressions to develop inferential procedures for multiple dependent sample alpha coefficients. Purpose of the Present Study

As suggested above, inferential procedures for CGs calculated from designs involving more than one facet have not been developed. In the absence of such developments, it is not possible to comment on the precision of a single coefficient or to make comparisons among two or more coefficients. Thus, the major focus of the present study was the development of inferential procedures for the CG estimate. The two-facet design, with both facets random, will be used to illustrate, in the next chapter, these developments. greatest detail. This design, referred to as Design VII (see Cronbach et al., 1972, Ch. 2), has a broad range of application to psychological and educational measurement problems; it is appropriate for studies involving multiple raters, observers, or observation periods. Further, this design is relatively uncomplicated, allowing the explication of the development of the inferential procedure without extensive notation. Extension of the generalizability approach to designs with more crossed facets is straightforward. 
The random facet model was chosen as the focus of this study because the author believes that most facets studied are, as asserted by Shavelson and Webb (1981) with the notion of exchangeability, sampled from a larger universe. In their view, if facet conditions can be exchanged with other potential conditions of the facet, the facet should be treated as random.

The present research used a method similar to that employed by Hakstian and Lind (1982) to develop an approximate variance expression for estimated CGs under different experimental design and sampling models. First, a normalizing transformation was applied to the expression for the sample coefficient. Then the delta method was used to derive the variance expressions. Details of these procedures are presented in the following chapter. 


\section{Chapter 2}

\section{Mathematical Development}

The development of the asymptotic variance expressions and their use in constructing confidence intervals will be illustrated for the fully-crossed two-facet design--Cronbach et al.'s (1972, p. 38) Design VII--with both facets random. This chapter begins with a discussion of the approximate distribution of the $C G$ and then proceeds with the application of a normalizing transformation. Following this step, the delta method is applied to the transformed coefficient to obtain the variance expression.

The development of variance expressions for other selected two-facet designs follows the same steps. The Approximate Distribution of the CG

The Schroeder et al. (1983) study discussed previously is an example of a Design VII G Study. In this case each of 71 inmates was rated on 22 items by each of two raters. This layout can be characterized as a between-within subjects balanced ANOVA design. The data for such a design are analysed as a three-way factorial design without replications (i.e., there is only one observation per cell). The linear model underlying these data is expressed as

$$
x_{p i r}=\mu+a_{p}+b_{i}+c_{r}+a b_{p i}+a c_{p r}+b c_{i r}+a b c_{p i r, \epsilon}
$$


where $\mu$ is the grand mean, $a_{p}$ is the effect due to person $p$, $b_{i}$ is the effect due to item $i$, and $c_{r}$ is the effect due to rater $r$. The remaining terms in the model represent the effects due to interactions among the factors. The epsilon in the subscript for the three-way interaction indicates that random error is confounded with this interaction. The formulas for the expected mean squares for the fully random ANOVA model are presented in Table 1. The mean squares and variance component estimates are presented in Table 2 . Throughout these derivations it is considered that the assumptions of the ANOVA model are tenable: independence of observations, homogeneity of variance, and underlying normal distribution.

The population CG for this design is

$$
\rho_{1}^{2}=\sigma_{p}^{2} /\left[\sigma_{p}^{2}+\sigma_{p i}^{2} / n_{i}+\sigma_{p r}^{2} / n_{r}+\sigma_{p i r, \epsilon}^{2} / n_{i} n_{I}\right]
$$

The subscript 1 is used to distinguish the CG for the fully random model from the CG for other models. The sample estimate for this quantity, expressed in terms of observed mean squares, is

$$
\hat{\rho}_{1}^{2}=1-\left[\left(\mathrm{MS}_{\mathrm{pi}}+\mathrm{MS} \mathrm{pr}_{\mathrm{pr}}-\mathrm{MS}_{\mathrm{pir}, \epsilon}\right) / \mathrm{MS} \mathrm{p}_{\mathrm{p}}\right] .
$$

First consider the numerator of the quantity $\left(1-\hat{\rho}_{1}^{2}\right)$,

$$
\mathrm{MS}_{\mathrm{pi}}+\mathrm{MS}_{\mathrm{pr}}-\mathrm{MS}_{\mathrm{pir}, \epsilon}
$$

This expression is a linear combination of independent 
Table 1

Expected Mean Squares for Design VII with both Facets Random

$\begin{array}{ll}E\left(M S_{p}\right) & =\sigma_{p i r, \epsilon}^{2}+n_{r} \sigma_{p i}^{2}+n_{i} \sigma_{p r}^{2}+n_{i} n_{r} \sigma_{p}^{2} \\ E\left(M S_{i}\right) & =\sigma_{p i r, \epsilon}^{2}+n_{r} \sigma_{p i}^{2}+n_{p} \sigma_{i r}^{2}+n_{p} n_{r} \sigma_{i}^{2} \\ E\left(M S_{r}\right) & =\sigma_{p i r, \epsilon}^{2}+n_{i} \sigma_{p r}^{2}+n_{p} \sigma_{i r}^{2}+n_{p} n_{i} \sigma_{r}^{2} \\ E\left(M S_{p i}\right) & =\sigma_{p i r, \epsilon}^{2}+n_{r} \sigma_{p i}^{2} \\ E\left(M S_{p r}\right) & =\sigma_{p i r, \epsilon}^{2}+n_{i} \sigma_{p r}^{2} \\ E\left(M S_{i r}\right) & =\sigma_{p i r, \epsilon}^{2}+n_{p} \sigma_{i r}^{2} \\ E\left(M S_{p i r, \epsilon}\right) & =\sigma_{p i r, \epsilon}^{2}\end{array}$


Table 2

Analysis of Variance Results and Estimated Variance Components for Psychopathy Data

\begin{tabular}{lrrrr}
\hline Source & Sum of Squares & df & Mean Square & $\hat{\sigma}^{2}$ \\
\hline Persons & 363.4225 & 70 & 5.1918 & .0015 \\
Items & 115.4882 & 21 & 5.4994 & .0311 \\
Raters & .7686 & 1 & .7686 & .0001 \\
P X I & 1021.9437 & 1470 & .6952 & .2604 \\
P X R & 14.2996 & 70 & .2043 & .0014 \\
I X R & 11.9286 & 21 & .5680 & .0055 \\
P X I X R & 256.5032 & 1470 & .1745 & .1745 \\
\hline
\end{tabular}


variance estimates. The exact form of the distribution of such a combination is too complicated to be of practical utility (see Fleiss, 1971). However, Satterthwaite (1941, 1946) developed an approximation to the distribution of such a linear combination. If $g$ is a linear combination of variance estimates,

$$
g=a_{1} s_{1}^{2}+a_{2} s_{2}^{2}+\ldots+a_{k} s_{k}^{2}
$$

where the sample variance $s_{i}^{2}$ has expected value $\sigma_{i}^{2}, r_{i}$ degrees of freedom, and weighting factor $a_{i}$, then the quantity $\mathrm{rg} / \mathrm{E}(\mathrm{g})$ is approximately chi-square distributed with $r$ degrees of Ereedom,

$$
r=\frac{\left(a_{1} \sigma_{1}^{2}+a_{2} \sigma_{2}^{2}+\ldots+a_{k} \sigma_{k}^{2}\right)^{2}}{\left(a_{1} \sigma_{1}^{2}\right)^{2} / r_{1}+\left(a_{2} \sigma_{2}^{2}\right)^{2} / r_{2}+\ldots+\left(a_{k} \sigma_{k}^{2}\right)^{2} / r_{k}}
$$

(Satterthwaite, 1941).

Considering the denominator of $\left(1-\hat{\rho}_{1}^{2}\right)$, note that the quantity

$$
\left(n_{p}-1\right) M S_{p} / E\left(M S_{p}\right)
$$

is chi-square distributed with $n_{p}-1$ degrees of freedom. Hence, the quantity $\left(1-\hat{\rho}_{1}^{2}\right)$ is approximately $F$ distributed with degrees of freedom $\nu_{1}$ and $\nu_{2}$ given by 


$$
\begin{aligned}
& \left(M S_{p i}+M S_{p r}-M S_{p i r, \epsilon}\right)^{2} \\
& \nu_{1}= \\
& \frac{M_{p i}^{2}}{\left(n_{p}-1\right)\left(n_{i}-1\right)}+\frac{M_{p r}^{2}}{\left(n_{p}-1\right)\left(n_{r}-1\right)}+\frac{M S_{p i r, \epsilon}^{2}}{\left(n_{p}-1\right)\left(n_{i}-1\right)\left(n_{r}-1\right)}
\end{aligned}
$$

and

$$
\nu_{2}=\left(n_{p}-1\right)
$$

The Normalization of a Function of the CG

The distributional properties of the quantity $\left(1-\hat{\rho}_{1}^{2}\right)$ noted above could be used to develop an approximate confidence interval in a similar manner to the technique used by Feldt (1965). However, the development of a variance expression permits not only the setting of confidence intervals; it can also be used to develop inferential techniques for hypothesis testing. In this latter application, having a coefficient with an underlying normal distribution permits the application of a variety of statistical procedures based on normal theory. For this reason, Paulson's (1942) normalizing transformation was applied to a function of the CG.

Paulson (1942) extended Wilson and Hilferty's (1931) work on a normalizing transformation for $x^{2}$ variables. Paulson developed a very close normalization for F-distributed random variables,

$$
\left(c F^{1 / 3}-\mu\right) \sigma^{-1} \dot{N}(0,1),
$$


where

$$
\begin{aligned}
& \mu=1-2 /\left(9 \nu_{1}\right) \\
& \sigma^{2}=\left[2 /\left(9 \nu_{2}\right)\right] \mathrm{F}^{2 / 3}+2 /\left(9 \nu_{1}\right), \\
& c=1-2 /\left(9 \nu_{2}\right)
\end{aligned}
$$

and the quantities $\nu_{1}$ and $\nu_{2}$ are the degrees of freedom associated with the $F$ ratio. Hakstian and whalen (1976) used Paulson's transformation in their development of a test of significance for multiple independent alpha coefficients (to simplify the expression they set the $c$ term equal to one). Their empirical results indicated that application of Paulson's result yielded a test with good Type I error control.

The value of the $c$ term, which corrects for bias in the cube root transformed $F$ variate, was close to unity in the present investigation; it was set equal to one for all analyses. With the smallest value of $\nu_{2}\left(n_{p}=25\right)$ considered in the present study, the actual value of $c$ is .9907 . With the larger samples considered, the value of $c$ is even closer to unity. Further, preliminary analysis of the adequacy of the transformed CG with and without the scaling factor indicated that the inclusion of the factor had virtually no impact on the control of Type I error.

The Derivation of an Asymptotic Variance Expression for the $\underline{\mathrm{CG}}$

In the present research, the delta method (Rao, 1973, p. 387) was used to develop the asymptotic variance 
expression for the CG. The delta method is a technique for deriving an asymptotic variance expression for a statistic that is expressed as a function of statistics with a known covariance structure.

We begin with a vector, $t$, which contains unbiased and consistent estimates of the elements of the vector $\theta$. The statistics in the vector $t$ have known covariance matrix $\Sigma$. By asymptotic theory, the vector

$(n)^{1 / 2}(t-\theta) \dot{M V N}(0, \Sigma)$, where $n$ is the sample size. The statistic $f(t)=\left(1-\hat{\rho}_{1}^{2}\right)^{1 / 3}$ estimates $f(\theta)$. By the delta method,

$$
[f(t)-f(\theta)] \dot{\sim} N\left(0, \sigma^{2}\right)
$$

where $\sigma^{2}=\phi^{\prime} \Sigma \phi$. The vector $\phi$ contains the partial derivatives, $\partial f(t) /\left.\partial t\right|_{t=\theta}$. Thus, $\sigma^{2}$ is the asymptotic variance of the statistic $f(t)$; in practice, the value of the variance is estimated by substituting sample values for parameter values in the variance expression.

In the present application of the delta method, the vector $t$ contains the four mean squares which compose the sample CG:

$$
t^{\prime}=\left(\mathrm{MS}_{\mathrm{p}},{ }_{\mathrm{MS}}, \mathrm{MS}_{\mathrm{pr}}, \mathrm{MS}_{\mathrm{pir}, \epsilon}\right) \cdot
$$

These are the unbiased and consistent estimates of the expected mean squares contained in $\theta$. The quantity 
$f(t)=\left(1-\hat{\rho}_{1}^{2}\right)^{1 / 3}$ is the consistent estimator of

$f(\theta)=\left(1-\rho_{1}^{2}\right)^{1 / 3}$. The matrix $\Sigma$ contains the variances and covariances of the four mean squares. In a balanced ANOVA

design the mean squares are independent; thus, $\Sigma$ is a diagonal matrix. A mean square is the variance of a normally distributed random variable. The variance of the sample mean square is

$$
\operatorname{Var}(M S)=2[E(M S)]^{2} / \nu,
$$

where $\nu$ is the degrees of freedom associated with the mean square (Scheffé, 1959, Ch. 7). In this application, the matrix $\Sigma$ is:

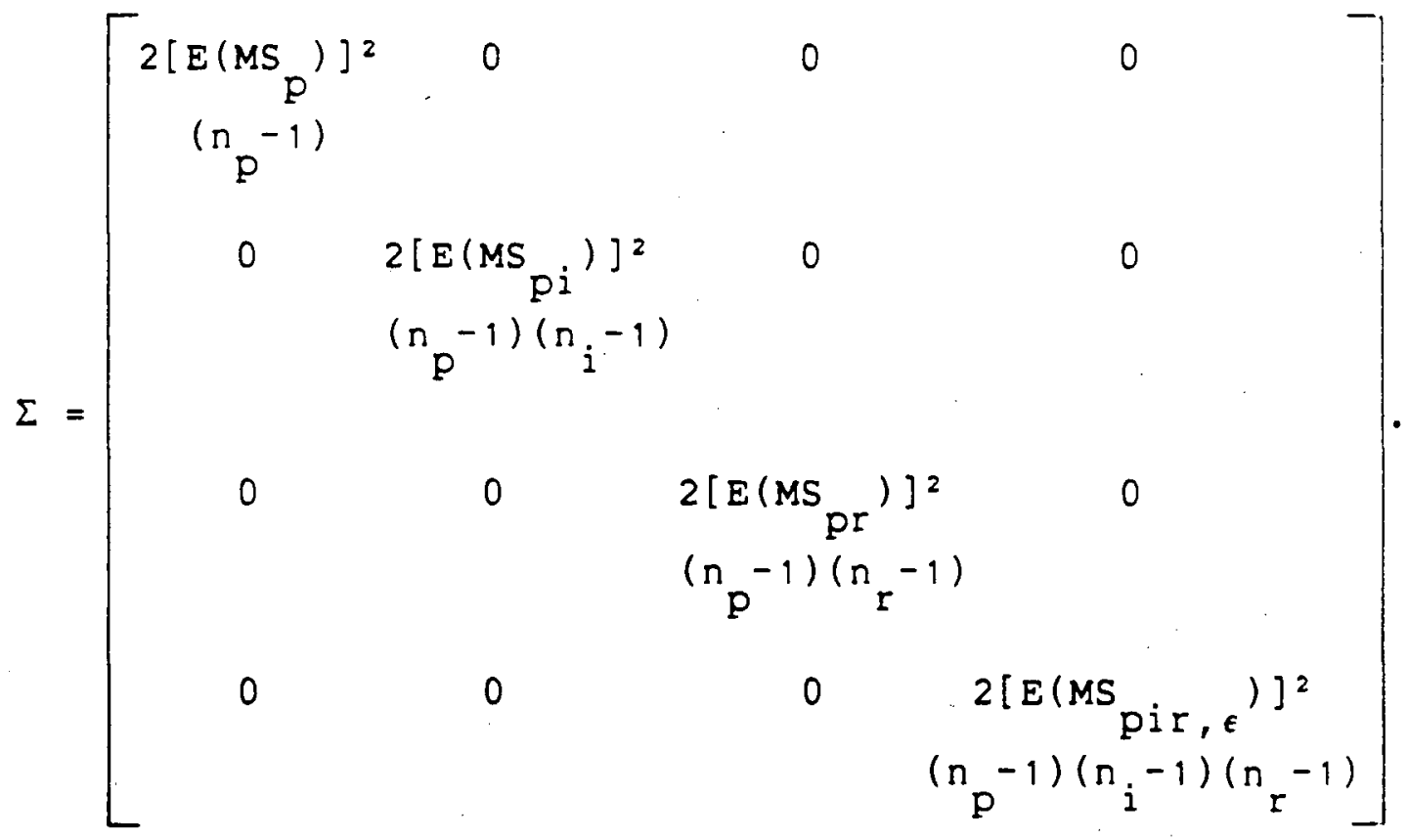

The partial derivatives of $f(t)$ contained in $\phi$ are

$$
\partial f(t) /\left.\partial M S_{p}\right|_{t=\theta}=1 / 3\left(1-\rho_{1}^{2}\right)^{1 / 3} / E\left(M S_{p}\right)
$$




$$
\begin{aligned}
& \partial f(t) /\left.\partial M S_{p i}\right|_{t=\theta}=1 / 3\left(1-\rho_{1}^{2}\right)^{-2 / 3} / E\left(M_{p}\right) \\
& \partial f(t) /\left.\partial M S_{p r}\right|_{t=\theta}=1 / 3\left(1-\rho_{1}^{2}\right)^{-2 / 3} / E\left(M_{p}\right) \\
& \partial f(t) /\left.\partial M S_{p i r, \epsilon}\right|_{t=\theta}=-1 / 3\left(1-\rho_{1}^{2}\right)^{-2 / 3} / E\left(M_{p}\right) .
\end{aligned}
$$

The asymptotic variance of the the transformed CG is given by the quantity

$$
\begin{aligned}
\operatorname{Var}\left(1-\hat{\rho}_{1}^{2}\right)^{1 / 3} & =\phi^{\prime} \Sigma_{\phi} \\
& =\frac{2\left(1-\rho_{1}^{2}\right)^{-4 / 3}}{9\left(n_{p}^{-1}\right)}\left\{\left(1-\rho_{1}^{2}\right)^{2}\right. \\
& +\frac{\left[E\left(M S_{p i}\right)\right]^{2}}{\left[E\left(M_{p}\right)\right]^{2}\left(n_{i}^{-1}\right)}+\frac{\left[E\left(M_{p r}\right)\right]^{2}}{\left[E\left(M_{p}\right)\right]^{2}\left(n_{r}^{-1}\right)} \\
& \left.+\frac{\left[E \left(M S_{p i r} \epsilon\right.\right.}{\left[E\left(M S_{p}\right)\right]^{2}\left(n_{i}-1\right)\left(n_{r}-1\right)}\right\}
\end{aligned}
$$

In practice, where the expected mean squares are not known, their unbiased estimates, the mean squares are substituted. The sample coefficient, $\hat{\rho}_{1}^{2}$, is substituted for the population value. This sample variance estimate is denoted $\widehat{\operatorname{Var}}\left(1-\hat{\rho}_{1}^{2}\right)^{1 / 3}$.

Variance Expressions for CGs Arising from other Experimental Designs

The delta method was used to derive asymptotic variance expressions for transformed coefficients arising from three other two-facet designs. In each case the CG was expressed 
in terms of mean squares, and the partial derivatives of the transformed coefficient were taken. The partial derivatives and the covariance matrix for the mean squares were then used to develop the asymptotic variance expression for the transformed CG.

Design VII with a fixed facet. In some situations all conditions of a particular facet may be included in the research design. We may have a design like that employed by Schroeder et al. where each person is evaluated on each item by each rater. It is possible that one of these facets cannot be considered random. This would be the case when, for example, specific tasks (i.e., items) had been developed for testing purposes or when raters have considerable expertise and cannot be viewed as exchangeable with other similar raters. In these cases the facet described would be considered fixed. This has two implications. First, the expressions for the expected mean squares are altered (see Winer, 1971, p. 344 for general formulas for the three-way factorial ANOVA). Second, the expression for the CG changes. The variance component for the interaction of the object of measurement with the fixed facet no longer is considered to be error variance; this variance is treated as universe score variance (Cronbach et al., 1972, Ch. 4). For example, in the following development the Item component is 
considered to be a fixed facet. The CG for Design VII with one fixed facet is

$$
\rho_{2}^{2}=\left[\sigma_{p}^{2}+\sigma_{p i}^{2} / n_{i}\right] /\left[\sigma_{p}^{2}+\sigma_{p i}^{2} / n_{i}+\left(\sigma_{p r}^{2} / n_{r}\right.\right.
$$

(6)

$$
\left.\left.+\sigma_{p i r, \epsilon}^{2} / n_{i} n_{r}\right)\right]
$$

where the subscript 2 indicates that the CG is for the fixed facet model. In terms of the observed mean squares, the CG is estimated by

$$
\hat{\rho}_{2}^{2}=1-\left[\left(\mathrm{MS}_{\mathrm{pr}}+\mathrm{MS}_{\mathrm{pir}, \epsilon}\right) /\left(\mathrm{MS}_{\mathrm{p}}+\mathrm{MS}_{\mathrm{pi}}\right)\right] .
$$

The corresponding asymptotic variance expression of $\left(1-\hat{\rho}_{2}^{2}\right)^{1 / 3}$, derived using the delta method, is

$$
\begin{aligned}
\operatorname{Var}\left(1-\hat{\rho}_{2}^{2}\right)^{1 / 3}= & \frac{2\left(1-\rho_{2}^{2}\right)^{-4 / 3}}{9\left[E\left(M S_{p}\right)+E\left(M S_{p i}\right)\right]^{2}\left(n_{p}^{-1}\right)} \\
& \left\{\left(1-\rho_{2}^{2}\right)^{2}\left[E\left(M S_{p}\right)\right]^{2}\right. \\
& +\frac{\left(1-\rho_{2}^{2}\right)^{2}\left[E\left(M S_{p i}\right)\right]^{2}}{\left(n_{i}^{-1)}\right.}+\frac{\left[E\left(M_{p r}\right)\right]^{2}}{\left(n_{r}^{-1)}\right.} \\
& +\frac{\left[E\left(M S_{p i r, \epsilon}\right)\right]^{2}}{\left(n_{i}^{-1)\left(n_{r}^{-1)}\right.}\right\}} .
\end{aligned}
$$

The sample estimate is denoted $\widehat{\operatorname{Var}}\left(1-\hat{\rho}_{2}^{2}\right)^{1 / 3}$. 
Design VII with a finite facet. Another variant on Design VII is a design in which the conditions of a facet are sampled at random from a finite universe. For example, a subset of four raters is drawn from a pool of 40 potential raters or four of twenty machines are selected for testing purposes. The interaction of the finite facet (here, raters) with the object of measurement is considered error variance--i.e., we wish to generalize beyond the raters used in the study to the finite population of potential from which they were randomly sampled. Thus, Raters becomes a finite facet. As in the case of the fixed facet design, the expressions for the expected mean squares are altered. The formula for the population CG, expressed in terms of variance components, is the same as that for the all facets raridom model (eq. 3 ).

In terms of observed mean squares, the estimated CG (the subscript 3 indicates CG for the finite model) can be expressed as

$\left.\hat{\rho}_{3}^{2}=\left[\left(\mathrm{MS}_{\mathrm{p}}-\mathrm{MS}_{\mathrm{pi}}-\mathrm{kMS_{pr }}+\mathrm{kMS_{pir } \epsilon}\right)_{\mathrm{p}}\right) /\left(\mathrm{MS}_{\mathrm{p}}+(1-k) M S_{p r}\right)\right]$ where $k=\left(1-n_{r} / N_{R}\right)$ and $n_{r} / N_{R}$, the sampling fraction, is the proportion of the population of conditions selected for inclusion in the study. As the fraction decreases, the value of $\hat{\rho}_{3}^{2}$ tends to the value of the CG for the fully random model. Application of the delta method yields the asymptotic variance expression 


$$
\begin{aligned}
& \operatorname{Var}\left(1-\hat{\rho}_{3}^{2}\right)^{1 / 3}=\frac{2\left(1-\rho_{3}^{2}\right)^{-4 / 3}}{9\left[\mathrm{E}\left(\mathrm{MS} \mathrm{p}_{\mathrm{p}}\right)+(k-1) \mathrm{E}\left(\mathrm{MS}_{\mathrm{pr}}\right)^{2}\left(\mathrm{n}_{\mathrm{p}}-1\right)\right.} \\
& \left\{\left(1-\rho_{3}^{2}\right)^{2}\left[E\left(M S_{p}\right)\right]^{2}+\frac{\left[E\left(M S_{p i}\right)\right]^{2}}{\left(n_{i}-1\right)}\right. \\
& \frac{+\left[\rho_{3}^{2}+k\left(1-\rho_{3}^{2}\right)\right]^{2}\left[E\left(M S_{p r}\right)\right]^{2}}{\left(n_{r}-1\right)} \\
& \left.+\frac{k^{2}[E(M S p i r, \epsilon}{\left(n_{i}-1\right)\left(n_{r}-1\right)}\right\}
\end{aligned}
$$

The sample variance estimate is denoted by $\widehat{\operatorname{Var}}\left(1-\hat{\rho}_{3}^{2}\right)^{1 / 3}$

\section{Design V-B with all facets random. In some}

circumstances, it is impractical or impossible to conduct a fully crossed D study. Sometimes a facet is naturally nested within another facet. In other circumstances it would be inefficient to conduct a fully crossed D study. As an example of a study with a nested facet, Kane and Brennan (1977) investigated the generalizability of course evaluation means. Classes were the objects of measurement. Students (i.e., Raters) are nested within the object of measurement. Another example of a nested design is a situation in which trainees' performance is rated on a series of dimensions by a panel of supervisors. In this example, each trainee has his/her own panel of supervisors. Both of these designs are examples of what cronbach et al. (1972, p. 38) termed Design V-B. The total observed score 
variance can be expressed as:

$$
\sigma^{2}\left(\mathrm{X}_{\mathrm{pir}}\right)=\sigma_{\mathrm{p}}^{2}+\sigma_{i}^{2}+\sigma_{\mathrm{r}: \mathrm{p}}^{2}+\sigma_{\mathrm{pi}}^{2}+\sigma_{\mathrm{i} r: \mathrm{p}, \epsilon}^{2}
$$

The variance component $\sigma_{r: p}^{2}$ confounds the sources of variance $\sigma_{r}^{2}$ and $\sigma_{\mathrm{pr}}^{2}$ from Design VII. Similarly, the residual variance, $\sigma_{i r: p, \epsilon}^{2}$, confounds $\sigma_{i r}^{2}$ and $\sigma_{\text {pir }}^{2} \epsilon$. From these relationships it is possible to estimate the magnitude of the CG for a V-B design from the variance component estimates derived from a Design VII $G$ study (the confounded variance components from Design VII are summed to yield the esimates for the V-B components).

The population value of the Design V-B CG with all facets random is:

$$
\rho_{4}^{2}=\sigma_{p}^{2} /\left[\sigma_{p}^{2}+\sigma_{p i}^{2} / n_{i}+\sigma_{r: p}^{2} / n_{r}+\sigma_{i r: p, \epsilon}^{2} / n_{i} n_{r}\right] .
$$

The subscript 4 indicates that the CG pertains to the Desigr $\mathrm{V}-\mathrm{B}$ model. In terms of mean squares, this quantity is estimated by:

$$
\hat{\rho}_{4}^{2}=1-\left[\left(M S_{p i}+M_{r: p}-M S_{i r: p, \epsilon}\right) / M S_{p}\right]
$$


The asymptotic variance expression for the normalized CG is:

$$
\begin{aligned}
\operatorname{Var}\left(1-\hat{\rho}_{4}^{2}\right)^{1 / 3}= & \frac{2\left(1-\rho_{4}^{2}\right)^{-4 / 3}}{9}\left\{\frac{\left(1-\rho_{4}^{2}\right)^{2}}{\left(n_{p}-1\right)}\right. \\
& +\frac{\left[E\left(M S_{p i}\right)\right]^{2}}{\left[E\left(M S_{p}\right)\right]^{2}\left(n_{p}-1\right)\left(n_{i}^{-1)}\right.} \\
& +\frac{\left[E\left(M S_{r: p}\right)\right]^{2}}{\left[E\left(M_{p}\right)\right]^{2} n_{p}\left(n_{r}^{-1}\right)} \\
& \left.+\frac{\left[E\left(M S_{i r: p, \epsilon}\right)\right]^{2}}{\left[E\left(M S_{p}\right)\right]^{2} n_{p}\left(n_{i}-1\right)\left(n_{r}-1\right)}\right\} .
\end{aligned}
$$

The sample estimate is denoted by $\widehat{\operatorname{Var}}\left(1-\hat{\rho}_{4}^{2}\right)^{1 / 3}$. 
Chapter 3

Method

Data Generation

Data were generated acccording to the model presented in Equation (2) for the three Design VII conditions. Each observation was composed of seven independent components with an underlying normal distribution.

The model for any given observation can be expressed as:

(7) $\quad x_{p i r}=x_{p}+x_{i}+x_{r}+x_{p i}+x_{p r}+x_{i r}+x_{p i r, \epsilon}$. The quantities on the right hand side of the equation are deviation scores. For Person $p$, an observation $x_{p}$ was generated from a $\mathrm{N}\left(0, \sigma_{\mathrm{p}}^{2}\right)$ distribution $\left(\mathrm{p}=1,2, \ldots, n_{p}\right)$. The $x_{i}\left(i=1,2, \ldots, n_{i}\right)$ and $x_{r}\left(r=1,2, \ldots, n_{r}\right)$ observations were generated in a similar manner. For Person $\mathrm{p}, n_{i}$ independent observations were generated from a $\mathrm{N}\left(0, \sigma_{\mathrm{pi}}^{2}\right)$ distribution $\left(p=1,2, \ldots, n_{p}\right)$. The $x_{p r}$ observations were generated similarly. For item $i,{ }_{r}$ independent observations were generated from a $N\left(0, \sigma_{i r}^{2}\right)$ distribution $(i=1$, $\left.2, \ldots, n_{i}\right)$. The final observations $x_{p i r, \epsilon}$ were generated for each person by generating $n_{i}$ times $n_{r}$ random observations from a $N\left(0, \sigma_{\text {pir, }}^{2}\right)$ distribution. This process yields a three dimensional Persons by Items by Raters data array as shown in Figure 1. 


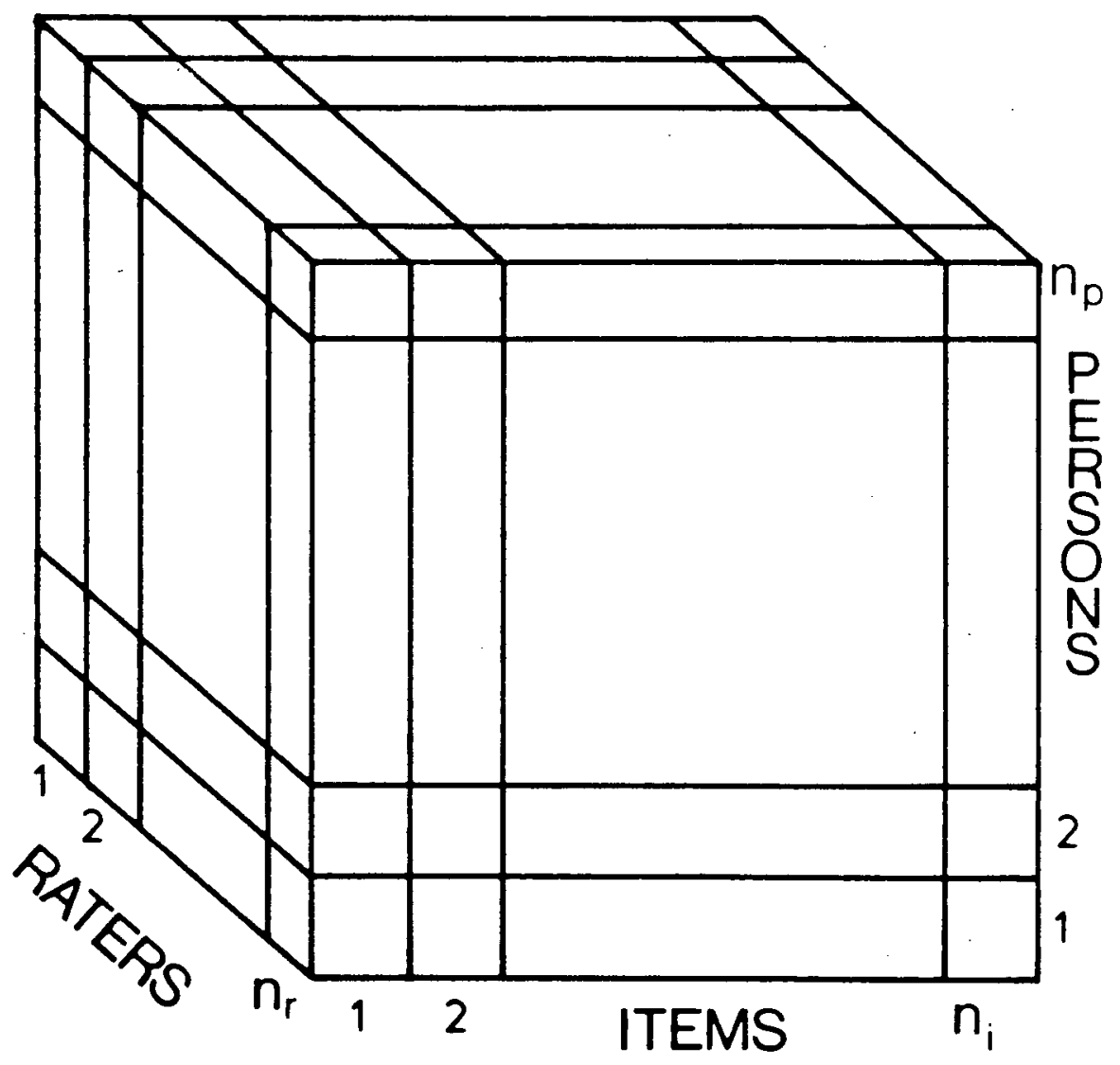

Figure 1. Layout of data for Design VII Analysis of Variance 
The normal variates were generated with the RANDN generator implemented on the University of British Columbia's Amdahl 580 computer. The RANDN algorithm begins by generating observations from a uniform $(0,1)$ distribution. These observations are transformed to normally distributed $(0,1)$ random samples by application of Marsaglia's rectangular wedge-tail method (Knuth, 1968). For the present study the observed score components were given the desired variance by multiplying them by the square root of the population variance component (see below).

The mean square components for the three-way ANOVA were then calculated from the data with a double precision Fortran routine. These components were used to calculate the sample CG and the estimated variance of the transformed coefficient. The procedure for generating the data for the Design V-B model differed from this method only in that five rather than seven variance components were involved in the expression for total variance. Analysis of these data yields five mean square components.

Overview of the study for Design VII with Both Facets Random

As previously noted, a larger number of situations were examined for Design VII with both facets random. A total of 81 combinations of facet conditions and sample size were were investigated for this model. Three sample sizes $\left(n_{p}=25,75,150\right)$, three conditions of Items $\left(n_{i}=10,20\right.$, 30), three conditions of Raters $\left(n_{r}=2,5,8\right)$, and three 
values of the population CG $\left(\rho^{2}=.5, .7, .9\right)$ were combined in a fully crossed factorial design. Population CGs were constructed by specifying suitable variance component values. For the 81 conditions the following values were held constant: $\sigma_{p}^{2}=1, \sigma_{i}^{2}=.6, \sigma_{r}^{2}=.5, \sigma_{i r}^{2}=.4$, and $\sigma_{\text {pir, } \epsilon}^{2}=.3$. The values of $\sigma_{i}^{2}, \sigma_{r}^{2}$, and $\sigma_{i r}^{2}$ do not enter into the computation of the mean squares used to construct the CG estimate for any of the situations examined in this study. Thus, the values for these variances were chosen arbitrarily. The values of $\sigma_{\mathrm{pi}}^{2}$ and $\sigma_{\mathrm{pr}}^{2}$ were set equal, and this value was determined to give the desired population CG for a particular condition. Preliminary simulations in which the magnitude of the population variance components were varied indicated that the magnitudes of the components have little if any impact on Type I error control. Three Other Models

The Design VII fixed facet and the Design V-B models were each investigated in nine combinations of condition and sample sizes. Three sets of values $\left(n_{p}=25, n_{i}=20\right.$, $\left.n_{r}=8\right),\left(n_{p}=75, n_{i}=30, n_{r}=2\right)$, and $\left(n_{p}=150, n_{i}=10\right.$, $\left.n_{r}=5\right)$ were crossed with the same three population CG values used above. The sets of values were chosen such that each set contained a low, a moderate, and a high value. For the finite model, two population sampling fractions were investigated: $n_{r} / N_{R}=.10$ and .20 . These values were crossed with the sets of conditions used with the Design VII fixed facet model and the Design V-B models, yielding 18 
conditions.

The data generation method for the variants on Design VII (finite and fixed facet models) differed somewhat from that used for the random facets model. For each set of conditions, population mean square terms were constructed that would yield the desired value of the CG for the model under study. Note that the computational procedures and the resultant mean squares are the same for a given set of data, regardless of which ANOVA model (fixed, random, or finite) is appropriate for the data. Variance component estimates are calculated from the mean squares according to the assumed underlying model. To generate data with the required population mean square values, the population mean squares were treated as if they had arisen from the fully random Design VII model. The random effects variance components were used to generate observations that were summed according to the model presented in Equation 7. Analysis of the resulting data gave $r$ ise to mean squares that behaved like sample estimates of the population mean squares appropriate to the model under study.

For example, consider the case where $\rho_{2}^{2}=.5$ for the Design VII model with the Item facet fixed and ${ }_{p}=25, n_{i}=$ 20 , and $n_{r}=8$, and the population expected mean squares $E\left(\right.$ MS $\left._{p}\right)=99.96, E\left(\right.$ MS $\left._{p i}\right)=5, E\left(\right.$ MS $\left._{p r}\right)=49.48$, and $E$ (MS $p i r, \epsilon$ ) $=3$. Solving for the variance components from Equations $8 a$ through $8 \mathrm{~d}$ gives the variance components for the Design VII 
model with the item facet fixed: $\sigma_{\mathrm{p}}^{2}=.3155, \sigma_{\mathrm{pi}}^{2}=.25$, $\sigma_{p r}^{2}=2.474$, and $\sigma_{p i r, \epsilon}^{2}=3$. Substituting these values into Equation 6 gives the desired value of $\rho_{2}^{2}=.5$.

$$
\begin{aligned}
& E\left(M_{p}\right)=n_{i} \sigma_{p r}^{2}+n_{i} n_{r} \sigma_{p}^{2} \\
& E\left(M{ }_{p i}\right)=\sigma_{p i r}^{2} \epsilon+n_{I} \sigma_{p i}^{2} \\
& E\left(M{ }_{p r}\right)=n_{i} \sigma_{p r}^{2} \\
& E\left(M P_{p i r, \epsilon}\right)=\sigma_{p i r, \epsilon}^{2}
\end{aligned}
$$

Note, however, that the $\sigma^{2}$ values generally are not equal to the values that would be calculated if a fully random effects model were assumed to underlie the data. In order to use the Equation 7 model for data generation it was necessary to determine the population variance components for the Design VII fully random model. These component values are then used to generate data in the same manner as used for the Design VII fully random model. The resultant mean square estimates behave as if they were sampled from a population with the required mean square values. The variance components used to generate the data for Design VII with a fixed facet, with a finite facet, and Design V-B are presented in Appendix A.

\section{A Special Condition: Zero Variance Components}

In all of the above conditions, the sample CG was calculated from the variance component estimates computed from observed mean squares. Any variance component estimate that involves subtraction of mean square estimates can yield a negative value. Negative estimates occur, for example, 
when the value of MS pir, $\epsilon$ is greater than the value of $\mathrm{MS}_{\mathrm{pi}}$ or $\mathrm{MS}_{\mathrm{pr}}$. Cronbach et al. (1972, p. 57) suggested that negative variance estimates are due to sampling error and recommended that such components be set equal to zero--their likely population value. These zero values then should be used in the estimate of higher variance components (e.g., if $\hat{\sigma}_{\mathrm{pi}}^{2}$ is set to zero, the estimate of $\sigma_{\mathrm{pi}}^{2}$ in the formula for $\sigma_{p}^{2}$ should also be set equal to zerol. This zero substitution results in a CG value that is lower than that obtained from the usual mean square formula. The zero substitution effectively deletes mean square terms from the CG expression. For example, in the case where the $\hat{o}_{\mathrm{pi}}^{2}$ estimate is negative (and is replaced by a zero value) the formula for the estimated CG, expressed in terms of mean squares, becomes:

$$
\hat{\rho}_{1}^{2}=\left(M S_{p}-M S_{p r}\right) / M S_{p}
$$

The estimate of the variance of the transformed coefficient thus also changes from that calculated when the zero estimate is ignored.

In the present study, negative variance estimates were dealt with two ways. First, the CG and the estimated variance were calculated from the mean squares without any adjustment for negative values. Second, the CG was calculated with adjustment made for negative estimates. The formula for the variance estimate was also adjusted to allow for the deletion of mean square terms in the estimated $C G$. 
These two treatments of zero components were compared in a series of 12 combinations of parameter values and numbers of conditions. Eight conditions used values of $n_{p}=25$, $n_{i}=10, n_{r}=2$ with CG values of .5 and .9. The remaining four conditions used values of $n_{p}=75, n_{i}=20, n_{r}=5$ for the same CG values. In half of these conditions $\sigma_{p i}^{2}$ was set to zero; in the other half $\sigma_{\mathrm{pr}}^{2}$ was set to zero. The population variance components used in this portion of the study are presented in Appendix A. The purpose of this investigation was to determine which, if either, treatment of zero components performed better when applied in conjunction with the inferential procedure proposed in this thesis.

\section{A Special Condition: Dichotomous Data}

The conditions described to this point all involve continuous dependent random variables. In many instances where persons are rating themselves or are being tested, the items cannot be viewed as continuous. Many personality inventories are composed of true-false items; most achievement and aptitude test items are scored as correct or incorrect. Because of the prevalence of this type of item in psychological tests, a set of conditions in which the individual item scores were dichotomous were investigated. The data, based on the Design VII random facets model, were generated in the same manner as in the continuous data case. The observations were then assigned the value 1 if they were greater than or equal to the population mean of zero and the 
value 0 if they were less than the mean. This procedure yields dichotomous items with a population difficulty level of .5. For this set of analyses, eight conditions were examined: $\left(n_{p}=25, n_{i}=10, n_{r}=2\right)$ and $\left(n_{p}=75, n_{i}=20\right.$, $\left.{ }_{r}=5\right)$ for each of the three values of the CG for continuous data $\left(\rho^{2}=.5, .7, .9\right)$ and $\left(n_{p}=25, n_{i}=10\right.$, $\left.n_{r}=5\right)$ and for two values of the CG $\left(\rho^{2}=.5, .9\right)$.

Dichotomizing the data alters the value of the population CG. Because of the complicated relationship between the population variance components for the continuous and dichotomized data, and the absence of a mathematically tractable analytic adjustment for dichotomization, it was necessary to estimate the population CG values empirically. Separate runs were conducted to provide estimates of the parameter values for the dichotomized conditions. For the conditions with $n_{p}=25$, 10,000 replications were simulated; 5,000 replications were run for conditions with $n_{p}=75$. These values were then used as the population values for the test statistic. Testing the Adequacy of the Asymptotic Variance Expression

For each of the conditions outlined above, and summarized in Table 3, 2,500 replications of the design were simulated. In each replication the transformed sample CG and the variance estimate were computed. The test statistic

$$
k=\left[\left(1-\hat{\rho}^{2}\right)^{1 / 3}-\left(1-\rho^{2}\right)^{1 / 3}\right] /\left[\widehat{\operatorname{Var}}\left(1-\hat{\rho}^{2}\right)\right]^{1 / 2}
$$


Table 3

Summary of Designs and Conditions for the Empirical Investigation

1. Design VII--Both Facets Random

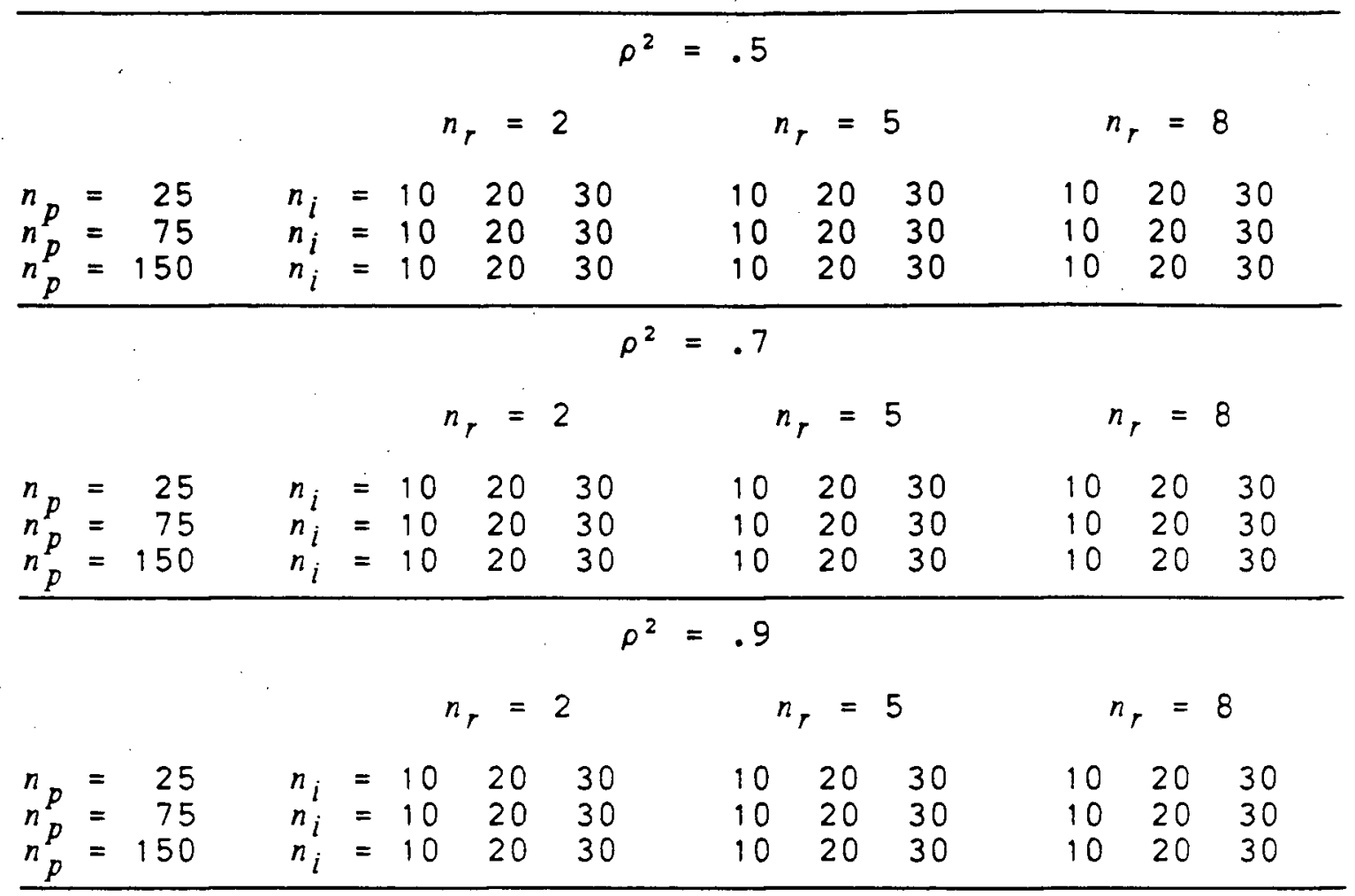


Table 3 (continued)

2. Design VII--Item Facet Fixed

\begin{tabular}{rrrrrrrrrr}
\hline \multicolumn{3}{c}{$\rho^{2}=.5$} & \multicolumn{3}{c}{$\rho^{2}=.7$} & \multicolumn{2}{c}{$\rho^{2}=.9$} \\
\hline$n_{p}$ & $n_{i}$ & $n_{r}$ & $n_{p}$ & $n_{i}$ & $n_{r}$ & $n_{p}$ & $n_{i}$ & $n_{r}$ \\
25 & 20 & 8 & 25 & 20 & 8 & 25 & 20 & 8 \\
75 & 30 & 2 & 75 & 30 & 2 & 75 & 30 & 2 \\
150 & 10 & 5 & 150 & 10 & 5 & 150 & 10 & 5 \\
\hline
\end{tabular}

3. Design VII--Rater Facet Finite

\begin{tabular}{|c|c|c|c|c|c|c|c|c|c|}
\hline \multicolumn{2}{|c|}{$\rho^{2}=$} & \multirow[t]{2}{*}{.5} & \multicolumn{2}{|r|}{$\rho^{2}$} & \multicolumn{2}{|l|}{.7} & \multicolumn{2}{|c|}{$\rho^{2}=$} & \multirow[t]{2}{*}{.9} \\
\hline & & & Sampling & Fract & ion & $=$ & .10 & & \\
\hline${ }^{n} p$ & $n_{i}$ & $n_{r}$ & ${ }^{n} p$ & $n_{i}$ & $n_{r}$ & & ${ }^{n} p$ & $n_{i}$ & $n_{r}$ \\
\hline $\begin{array}{r}25 \\
75 \\
150\end{array}$ & $\begin{array}{l}20 \\
30 \\
10\end{array}$ & $\begin{array}{l}8 \\
2 \\
5\end{array}$ & $\begin{array}{r}25 \\
75 \\
150\end{array}$ & $\begin{array}{l}20 \\
30 \\
10\end{array}$ & $\begin{array}{l}8 \\
2 \\
5\end{array}$ & & $\begin{array}{r}25 \\
75 \\
150\end{array}$ & $\begin{array}{l}20 \\
30 \\
10\end{array}$ & $\begin{array}{l}8 \\
2 \\
5\end{array}$ \\
\hline & & & Sampling & Fract & ion & $=$ & .20 & & \\
\hline${ }^{n} p$ & $n_{i}$ & $n_{r}$ & $n_{p}$ & $n_{i}$ & $n_{r}$ & & ${ }^{n} p$ & $n_{i}$ & $n_{r}$ \\
\hline $\begin{array}{r}25 \\
75 \\
150\end{array}$ & $\begin{array}{l}20 \\
30 \\
10\end{array}$ & $\begin{array}{l}8 \\
2 \\
5\end{array}$ & $\begin{array}{r}25 \\
75 \\
150\end{array}$ & $\begin{array}{l}20 \\
30 \\
10\end{array}$ & $\begin{array}{l}8 \\
2 \\
5\end{array}$ & & $\begin{array}{r}25 \\
75 \\
150\end{array}$ & $\begin{array}{l}20 \\
30 \\
10\end{array}$ & $\begin{array}{l}8 \\
2 \\
5\end{array}$ \\
\hline
\end{tabular}

4. Design V-B Both Facets Random

\begin{tabular}{rrrrrrrrrr}
\hline & $\rho^{2}=$ & \multicolumn{4}{c}{$\rho^{2}=.7$} & & $\rho^{2}=.9$ \\
\hline$n_{p}$ & $n_{i}$ & $n_{r}$ & $n_{p}$ & $n_{i}$ & $n_{r}$ & $n_{p}$ & $n_{i}$ & $n_{r}$ \\
25 & 20 & 8 & 25 & 20 & 8 & 25 & 20 & 8 \\
75 & 30 & 2 & 75 & 30 & 2 & 75 & 30 & 2 \\
150 & 10 & 5 & 150 & 10 & 5 & 150 & 10 & 5 \\
\hline
\end{tabular}


Table 3 (continued)

5. Design VII with Zero Variance Component

\begin{tabular}{|c|c|c|c|c|c|c|c|c|c|c|c|}
\hline \multicolumn{6}{|c|}{$\sigma_{p i}^{2}=0$} & \multicolumn{6}{|c|}{$\sigma_{\mathrm{pr}}^{2}=0$} \\
\hline & $2=.5$ & 5 & f & $2=$ & .9 & & $=$ & .5 & $\rho$ & $2=$ & .9 \\
\hline${ }^{n} p$ & $n_{i} \quad n_{1}$ & $r$ & $n_{p}$ & $n_{i}$ & $n_{r}$ & ${ }^{n} p$ & $n_{i}$ & $n_{r}$ & ${ }^{n} p$ & $n_{i}$ & $n_{r}$ \\
\hline $\begin{array}{l}25 \\
75\end{array}$ & $\begin{array}{l}10 \\
20\end{array}$ & $\begin{array}{l}2 \\
5\end{array}$ & $\begin{array}{l}25 \\
75\end{array}$ & $\begin{array}{l}10 \\
20\end{array}$ & $\begin{array}{l}2 \\
5\end{array}$ & $\begin{array}{l}25 \\
75\end{array}$ & $\begin{array}{l}10 \\
20\end{array}$ & $\begin{array}{l}2 \\
5\end{array}$ & $\begin{array}{l}25 \\
75\end{array}$ & $\begin{array}{l}10 \\
20\end{array}$ & $\begin{array}{l}2 \\
5\end{array}$ \\
\hline
\end{tabular}

6. Design VII with Dichotomous Data

\begin{tabular}{ccccccccccc}
\hline \multicolumn{3}{c}{$\rho^{2}=$} & \multicolumn{1}{c}{$\rho^{2}=.7$} & & \multicolumn{2}{c}{$\rho^{2}=.9$} \\
\hline$n_{p}$ & $n_{i}$ & $n_{r}$ & $n_{p}$ & $n_{i}$ & $n_{r}$ & & $n_{p}$ & $n_{i}$ & $n_{r}$ \\
25 & 20 & 8 & 25 & 20 & 8 & & 25 & 20 & 8 \\
25 & 10 & 5 & & & & 25 & 10 & 5 \\
75 & 20 & 5 & 75 & 20 & 5 & & 75 & 20 & 5 \\
\hline
\end{tabular}


was calculated; the resulting value of $k$ was compared to $s i x$ points of the normal distribution corresponding to the $a=.10, .05, .01$ two-tailed levels of significance. 
Chapter 4

Results

In this section the results of the Monte Carlo study are presented. These results are presented for the different experimental designs in the order in which the variance expressions were developed in Chapter Two.

\section{Design VII with Both Facets Random}

The results of the study were examined for main effects due to the factors: sample size, conditions of Items, conditions of Raters, and population coefficient values. Additionally, the results were examined for the presence of two-way interactions among these factors. Results for the 81 conditions of Design VII with both facets random are presented in Tables 4,5 , and 6 . These results are organized according to the value of the population coefficient of generalizability. Summary statistics-mean proportions and overall chi-square values--for the main effects are presented in Table 7. No evidence of two-way interaction in the results was seen.

The overall chi-square goodness of fit statistic (with 27 degrees of freedom) is included in these tables to assist the reader in evaluating the results. The statistic is

$$
x^{2}=2500 \sum_{i=1}^{27}\left(\hat{p}_{i}-p\right)^{2} /[\hat{p}(1-\hat{p})],
$$

or 


$$
x^{2}=2500 \sum_{i=1}^{27}\left(\hat{a}_{i}-a\right)^{2} /[\hat{a}(1-\hat{a})],
$$

where $\hat{p}_{i}$ is the empirical proportion for combination $i$ of sample size, facet conditions, and population $C G, \hat{a}_{i}$ is the empirical alpha level, and $\hat{p}$ is the mean of the $\hat{p}_{i}$ for a particular proportion averaged over 27 cases; $\hat{a}$ is defined similarly. The critical values of the chi-square distribution for the .05 and .01 significance levels are 40.11 and 46.96 respectively. For this statistic, the nominal alpha or proportion is subtracted from the empirical value and is divided by a consistent estimator of the population standard deviation, given a true null hypothesis. The resultant value is a normally distributed random variable. Squaring and summing these for the cases yields a chi-square random variable with 27 degrees of freedom. It should be noted that these tests are very powerful because of the large number of replications used to estimate the sample proportion. Therefore, large test statistic values, even though significant, are not necessarily indicative of poor fit. These values are included for comparative purposes.

The empirical alpha levels for the Design VII results are close to their corresponding nominal values. From Table 4 for $\rho_{1}^{2}=.5$ it can be seen that all 27 observed values fell within two binomial standard errors:

$$
2[(.10 \times .90) / 2500]^{1 / 2}=.012 \text {, }
$$

of the nominal alpha level of .10. For nominal alpha of .05 , 
Table 4

Proportion of the Standardized Estimates Falling below Selected Percentile Points of the Unit Normal Distribution and Actual Proportion of Type I Errors for $\rho^{2}=.50$ (Design VII, both Facets Random)

\begin{tabular}{|c|c|c|c|c|c|c|c|c|c|c|c|c|}
\hline$n_{p}$ & $n_{i}$ & $n_{r}$ & $p:$ & 005 & 025 & 050 & 950 & 975 & 995 & $a: 10$ & 05 & 01 \\
\hline 25 & 10 & 2 & & 013 & 038 & 060 & 960 & 981 & 997 & 100 & 057 & 016 \\
\hline 25 & 20 & 2 & & 014 & 038 & 062 & 966 & 985 & 1000 & 096 & 053 & 014 \\
\hline 25 & 30 & 2 & & 012 & 045 & 068 & 965 & 990 & 999 & 102 & 056 & 013 \\
\hline 75 & 10 & 2 & & 012 & 036 & 064 & 953 & 982 & 1000 & 110 & 055 & 012 \\
\hline 75 & 20 & 2 & & 009 & 032 & 060 & 9.54 & 980 & 998 & 106 & 052 & 011 \\
\hline 75 & 30 & 2 & & 010 & 039 & 066 & 959 & 984 & 998 & 106 & 055 & 012 \\
\hline 150 & 10 & 2 & & 009 & 029 & 055 & 955 & 978 & 998 & 100 & 051 & 011 \\
\hline 150 & 20 & 2 & & 004 & 029 & 052 & 956 & 978 & 997 & 095 & 051 & 007 \\
\hline 150 & 30 & 2 & & 007 & 027 & 052 & 958 & 981 & 998 & 094 & 046 & 009 \\
\hline 25 & 10 & 5 & & 004 & 022 & 045 & 944 & 974 & 997 & 101 & 049 & 007 \\
\hline 25 & 20 & 5 & & 007 & 025 & 044 & 940 & 971 & 993 & 104 & 054 & 014 \\
\hline 25 & 30 & 5 & & 006 & 025 & 042 & 944 & 976 & 995 & 098 & 048 & 011 \\
\hline 75 & 10 & 5 & & 004 & 027 & 049 & 942 & 972 & 993 & 107 & 055 & 011 \\
\hline 75 & 20 & 5 & & 005 & 024 & 044 & 942 & 972 & 996 & 102 & 053 & 009 \\
\hline 75 & 30 & 5 & & 008 & 027 & 053 & 940 & 975 & 996 & 112 & 052 & 012 \\
\hline 150 & 10 & 5 & & 004 & 024 & 053 & 948 & 975 & 996 & 106 & 049 & 008 \\
\hline 150 & 20 & 5 & & 005 & 023 & 049 & 945 & 973 & 994 & 104 & 050 & 011 \\
\hline 150 & 30 & 5 & & 005 & 022 & 044 & 946 & 972 & 995 & 099 & 050 & 010 \\
\hline 25 & 10 & 8 & & 004 & 019 & 036 & 941 & 972 & 993 & 095 & 047 & 011 \\
\hline 25 & 20 & 8 & & 004 & 028 & 046 & 948 & 972 & 995 & 098 & 056 & 009 \\
\hline 25 & 30 & 8 & & 004 & 022 & 040 & 946 & 972 & 997 & 093 & 050 & 007 \\
\hline 75 & 10 & 8 & & 003 & 024 & 048 & 945 & 974 & 996 & 103 & 050 & 007 \\
\hline 75 & 20 & 8 & & 004 & 020 & 041 & 946 & 975 & 996 & 095 & 046 & 008 \\
\hline 75 & 30 & 8 & & 004 & 021 & 044 & 950 & 978 & 996 & 094 & 043 & 008 \\
\hline 150 & 10 & 8 & & 002 & 017 & 042 & 954 & 976 & 997 & 089 & 041 & 005 \\
\hline 150 & 20 & 8 & & 003 & 021 & 048 & 949 & 975 & 996 & 099 & 046 & 007 \\
\hline 150 & 30 & 8 & & 005 & 021 & 050 & 947 & 978 & 997 & 103 & 043 & 008 \\
\hline Mean & or & $\hat{a}_{i}$ & & 006 & 027 & 050 & 950 & 977 & 996 & 100 & 050 & 010 \\
\hline
\end{tabular}

$\begin{array}{lllllllll}144.1 & 131.1 & 91.7 & 75.8 & 71.0 & 91.6 & 23.0 & 25.2 & 45.1\end{array}$

Note. Decimal points omitted in alphas and proportions.

The standardized estimate is the value

$\left[\left(1-\hat{\rho}^{2}\right)^{1 / 3}-\left(1-\rho^{2}\right)^{1 / 3}\right] /\left[\widehat{\operatorname{Var}}\left(1-\hat{\rho}^{2}\right)^{1 / 3}\right]^{1 / 2}$. 
Table 5

Proportion of the Standardized Estimates Falling below Selected Percentile Points of the Unit Normal Distribution and Actual Proportion of Type I Errors for $\rho^{2}=.70$ (Design VII, both Facets Random)

\begin{tabular}{rlllllllllllll}
\hline $\mathrm{n}_{\mathrm{p}}$ & $\mathrm{n}_{\mathrm{i}}$ & $\mathrm{n}_{I}$ & $\mathrm{p}:$ & 005 & 025 & 050 & 950 & 975 & 995 & $\mathrm{a}: 10$ & 05 & 01 \\
\hline 25 & 10 & 2 & 013 & 045 & 070 & 964 & 986 & 998 & 106 & 058 & 015 \\
25 & 20 & 2 & 014 & 043 & 073 & 958 & 982 & 996 & 114 & 060 & 018 \\
25 & 30 & 2 & 018 & 046 & 070 & 956 & 979 & 999 & 114 & 067 & 019 \\
75 & 10 & 2 & 006 & 038 & 065 & 963 & 984 & 998 & 102 & 054 & 008 \\
75 & 20 & 2 & 006 & 024 & 055 & 962 & 983 & 998 & 093 & 041 & 008 \\
75 & 30 & 2 & 014 & 042 & 070 & 958 & 982 & 997 & 112 & 060 & 017 \\
150 & 10 & 2 & 005 & 031 & 062 & 959 & 984 & 996 & 103 & 048 & 009 \\
150 & 20 & 2 & 007 & 031 & 053 & 964 & 980 & 996 & 090 & 050 & 011 \\
150 & 30 & 2 & 008 & 030 & 054 & 950 & 974 & 995 & 104 & 056 & 013 \\
25 & 10 & 5 & 004 & 022 & 040 & 946 & 973 & 994 & 094 & 049 & 010 \\
25 & 20 & 5 & 004 & 020 & 044 & 951 & 977 & 997 & 092 & 043 & 007 \\
25 & 30 & 5 & 005 & 022 & 045 & 937 & 971 & 994 & 108 & 051 & 011 \\
75 & 10 & 5 & 004 & 024 & 054 & 944 & 970 & 994 & 110 & 054 & 010 \\
75 & 20 & 5 & 004 & 025 & 048 & 951 & 974 & 998 & 097 & 052 & 006 \\
75 & 30 & 5 & 007 & 026 & 052 & 953 & 978 & 998 & 098 & 048 & 009 \\
150 & 10 & 5 & 003 & 021 & 047 & 952 & 974 & 994 & 095 & 048 & 009 \\
150 & 20 & 5 & 006 & 030 & 056 & 955 & 979 & 994 & 100 & 051 & 012 \\
150 & 30 & 5 & 005 & 025 & 044 & 945 & 973 & 994 & 100 & 052 & 011 \\
25 & 10 & 8 & 005 & 023 & 048 & 933 & 970 & 993 & 115 & 053 & 012 \\
25 & 20 & 8 & 005 & 022 & 046 & 939 & 972 & 996 & 107 & 050 & 009 \\
25 & 30 & 8 & 006 & 024 & 044 & 949 & 974 & 994 & 094 & 050 & 012 \\
75 & 10 & 8 & 006 & 023 & 043 & 951 & 974 & 994 & 092 & 049 & 012 \\
75 & 20 & 8 & 004 & 022 & 049 & 944 & 968 & 994 & 105 & 053 & 010 \\
75 & 30 & 8 & 003 & 021 & 047 & 943 & 976 & 997 & 104 & 045 & 006 \\
150 & 10 & 8 & 004 & 022 & 044 & 941 & 968 & 994 & 103 & 054 & 010 \\
150 & 20 & 8 & 004 & 025 & 050 & 944 & 974 & 996 & 107 & 051 & 008 \\
150 & 30 & 8 & 005 & 021 & 043 & 947 & 976 & 997 & 096 & 045 & 008 \\
& & & & & & & & & & & \\
Mean & $\mathrm{p}_{\mathrm{i}}$ or & $\hat{0} \mathrm{i}$ & 006 & 028 & 052 & 950 & 976 & 996 & 102 & 052 & 011 \\
& & & & & & & & & & & \\
$0 \mathrm{vera} 11 \mathrm{Chi}-\mathrm{square}(\mathrm{df}$ & $27)$ & & & & & & &
\end{tabular}

$\begin{array}{lllllllll}174.5 & 169.4 & 132.4 & 97.8 & 76.5 & 60.2 & 42.1 & 44.5 & 66.8\end{array}$

Note. Decimal points omitted in alphas and proportions.

The standardized estimate is the value

$\left[\left(1-\hat{\rho}^{2}\right)^{1 / 3}-\left(1-\rho^{2}\right)^{1 / 3}\right] /\left[\widehat{\operatorname{Var}}\left(1-\hat{\rho}^{2}\right)^{1 / 3}\right]^{1 / 2}$. 
Table 6

Proportion of the Standardized Estimates Falling below Selected Percentile Points of the Unit Normal Distribution and Actual Proportion of Type I Errors for $\rho^{2}=.90$ (Design VII, both Facets Random )

\begin{tabular}{|c|c|c|c|c|c|c|c|c|c|c|c|c|}
\hline$n_{p}$ & $\mathrm{n}_{\mathrm{i}}$ & $\mathrm{n}_{\mathrm{r}}$ & p: & 005 & 025 & 050 & 950 & 975 & 995 & $a: 10$ & 05 & 01 \\
\hline 25 & 10 & 2 & & 016 & 047 & 078 & 969 & 987 & 1000 & 109 & 060 & 016 \\
\hline 25 & 20 & 2 & & 014 & 043 & 074 & 962 & 986 & 999 & 113 & 058 & 015 \\
\hline 25 & 30 & 2 & & 018 & 054 & 084 & 964 & 986 & 1000 & 120 & 068 & 018 \\
\hline 75 & 10 & 2 & & 010 & 033 & 060 & 959 & 979 & 998 & 101 & 054 & 012 \\
\hline 75 & 20 & 2 & & 009 & 034 & 060 & 958 & 980 & 998 & 102 & 054 & 011 \\
\hline 75 & 30 & 2 & & 006 & 026 & 047 & 956 & 984 & 996 & 090 & 042 & 010 \\
\hline 150 & 10 & 2 & & 009 & 031 & 060 & 958 & 980 & 996 & 102 & 051 & 013 \\
\hline 150 & 20 & 2 & & 006 & 028 & 054 & 952 & 979 & 997 & 102 & 049 & 009 \\
\hline 150 & 30 & 2 & & 011 & 035 & 060 & 958 & 981 & 997 & 102 & 054 & 013 \\
\hline 25 & 10 & 5 & & 004 & 022 & 041 & 945 & 973 & 994 & 096 & 049 & 010 \\
\hline 25 & 20 & 5 & & 006 & 027 & 048 & 943 & 974 & 995 & 105 & 053 & 011 \\
\hline 25 & 30 & 5 & & 008 & 028 & 046 & 951 & 978 & 995 & 095 & 050 & 013 \\
\hline 75 & 10 & 5 & & 004 & 022 & 043 & 936 & 970 & 996 & 106 & 053 & 008 \\
\hline 75 & 20 & 5 & & 004 & 022 & 052 & 943 & 972 & 994 & 108 & 050 & 010 \\
\hline 75 & 30 & 5 & & 006 & 030 & 058 & 948 & 972 & 996 & 109 & 057 & 010 \\
\hline 150 & 10 & 5 & & 006 & 022 & 045 & 947 & 975 & 994 & 098 & 047 & 012 \\
\hline 150 & 20 & 5 & & 007 & 021 & 048 & 956 & 976 & 997 & 092 & 045 & 101 \\
\hline 150 & 30 & 5 & & 008 & 026 & 054 & 946 & 976 & 997 & 108 & 050 & 011 \\
\hline 25 & 10 & 8 & & 003 & 024 & 043 & 935 & 966 & 992 & 108 & 058 & 011 \\
\hline 25 & 20 & 8 & & 004 & 022 & 042 & 940 & 968 & 994 & 102 & 054 & 010 \\
\hline 25 & 30 & 8 & & 006 & 021 & 040 & 941 & 970 & 994 & 100 & 050 & 012 \\
\hline 75 & 10 & 8 & & 003 & 022 & 037 & 945 & 976 & 995 & 092 & 046 & 008 \\
\hline 75 & 20 & 8 & & 002 & 023 & 047 & 941 & 969 & 993 & 106 & 054 & 009 \\
\hline 75 & 30 & 8 & & 005 & 025 & 048 & 940 & 975 & 995 & 108 & 050 & 010 \\
\hline 150 & 10 & 8 & & 004 & 020 & 046 & 949 & 974 & 996 & 097 & 046 & 008 \\
\hline 150 & 20 & 8 & & 008 & 024 & 052 & 951 & 980 & 997 & 100 & 045 & 011 \\
\hline 150 & 30 & 8 & & 007 & 026 & 040 & 946 & 972 & 996 & 094 & 054 & 011 \\
\hline Mean & or & $\hat{a}_{i}$ & & 007 & 028 & 052 & 950 & 976 & 996. & 102 & 052 & 011 \\
\hline \multirow[t]{2}{*}{ Overa } & $\mathrm{I} \mathrm{Ch}$ & & & (aI & 27) & & & & & & & \\
\hline & & & & 9.2 & 192.0 & 188.4 & 106.3 & 95.4 & 80.3 & 38.4 & 43.2 & 40.9 \\
\hline
\end{tabular}

Note. Decimal points omitted in alphas and proportions.

The standardized estimate is the value

$\left[\left(1-\hat{\rho}^{2}\right)^{1 / 3}-\left(1-\rho^{2}\right)^{1 / 3}\right] /\left[\widehat{\operatorname{Var}}\left(1-\hat{\rho}^{2}\right)^{1 / 3}\right]^{1 / 2}$. 
Table 7

Mean Values and Overall Chi-square Values by Parameter Values and Facet Conditions (Design VII, both Facets Random) for Selected

Percentile Points of the Unit Normal Distribution and Three Levels of Type I Error

\begin{tabular}{|c|c|c|c|c|c|c|c|c|c|}
\hline $\mathrm{p}:$ & : 005 & 025 & 050 & 950 & 975 & 995 & $a: 10$ & 05 & 01 \\
\hline \multicolumn{10}{|c|}{ Coefficient Values } \\
\hline $\begin{array}{c}\rho^{2}=.5 \text { Mean } \\
x^{2}(27)\end{array}$ & $\begin{array}{c}006 \\
144.1\end{array}$ & $\begin{array}{c}027 \\
131.1\end{array}$ & $\begin{array}{l}050 \\
91.7\end{array}$ & $\begin{array}{l}950 \\
75.8\end{array}$ & $\begin{array}{l}977 \\
71.0\end{array}$ & $\begin{array}{l}996 \\
91.6\end{array}$ & $\begin{array}{l}100 \\
23.0\end{array}$ & $\begin{array}{l}050 \\
25.2\end{array}$ & $\begin{array}{r}010 \\
45.1\end{array}$ \\
\hline $\begin{array}{c}\rho^{2}=.7 \text { Mean } \\
x^{2}(27)\end{array}$ & $\begin{array}{l}006 \\
174.5\end{array}$ & $\begin{array}{c}028 \\
169.4\end{array}$ & $\begin{array}{c}052 \\
132.4\end{array}$ & $\begin{array}{l}950 \\
97.8\end{array}$ & $\begin{array}{l}976 \\
76.5\end{array}$ & $\begin{array}{l}996 \\
60.2\end{array}$ & $\begin{array}{l}102 \\
42.1\end{array}$ & $\begin{array}{l}052 \\
44.4\end{array}$ & $\begin{array}{l}011 \\
66.8\end{array}$ \\
\hline $\begin{array}{c}\rho^{2}=.9 \text { Mean } \\
\chi^{2}(27)\end{array}$ & $\begin{array}{c}007 \\
182.9\end{array}$ & $\begin{array}{l}028 \\
192.0\end{array}$ & $\begin{array}{c}052 \\
188.4\end{array}$ & $\begin{array}{c}950 \\
106.3\end{array}$ & $\begin{array}{l}976 \\
95.4\end{array}$ & $\begin{array}{l}996 \\
80.3\end{array}$ & $\begin{array}{l}102 \\
38.4\end{array}$ & $\begin{array}{l}052 \\
43.2\end{array}$ & $\begin{array}{l}011 \\
40.9\end{array}$ \\
\hline \multicolumn{10}{|c|}{ Number of Persons } \\
\hline $\begin{array}{l}25 \text { Mean } \\
x^{2}(27)\end{array}$ & $\begin{array}{c}008 \\
276.6\end{array}$ & $\begin{array}{c}030 \\
319.1\end{array}$ & $\begin{array}{c}052 \\
273.6\end{array}$ & $\begin{array}{c}950 \\
156.1\end{array}$ & $\begin{array}{c}976 \\
130.6\end{array}$ & $\begin{array}{c}996 \\
111.1\end{array}$ & $\begin{array}{l}103 \\
47.8\end{array}$ & $\begin{array}{l}054 \\
60.4\end{array}$ & $\begin{array}{l}012 \\
86.3\end{array}$ \\
\hline $\begin{array}{c}\mathrm{n}_{\mathrm{p}}=75 \quad \text { Mean } \\
x^{2}(27)\end{array}$ & $\begin{array}{l}006 \\
110.3\end{array}$ & $\begin{array}{c}027 \\
103.0\end{array}$ & $\begin{array}{l}052 \\
96.6\end{array}$ & $\begin{array}{l}949 \\
76.6\end{array}$ & $\begin{array}{l}976^{\prime} \\
68.3\end{array}$ & $\begin{array}{l}996 \\
78.4\end{array}$ & $\begin{array}{l}103 \\
36.2\end{array}$ & $\begin{array}{l}051 \\
31.1\end{array}$ & $\begin{array}{r}010 \\
34.5\end{array}$ \\
\hline $\begin{array}{c}n_{p}=150 \text { Mean } \\
x^{2}(27)\end{array}$ & $\begin{array}{l}006 \\
53.4\end{array}$ & $\begin{array}{l}025 \\
52.9\end{array}$ & $\begin{array}{l}050 \\
44.2\end{array}$ & $\begin{array}{l}951 \\
46.4\end{array}$ & $\begin{array}{l}976 \\
41.2\end{array}$ & $\begin{array}{l}996 \\
42.7\end{array}$ & $\begin{array}{l}099 \\
18.8\end{array}$ & $\begin{array}{l}049 \\
19.9\end{array}$ & $\begin{array}{l}010 \\
25.7\end{array}$ \\
\hline \multicolumn{10}{|c|}{ Number of Items } \\
\hline $\begin{array}{c}\mathrm{n}_{i}=10 \quad \text { Mean } \\
x^{2}(27)\end{array}$ & $\begin{array}{c}006 \\
162.1\end{array}$ & $\begin{array}{c}027 \\
162.1\end{array}$ & $\begin{array}{c}051 \\
152.6\end{array}$ & $\begin{array}{c}950 \\
115.8\end{array}$ & $\begin{array}{l}976 \\
87.4\end{array}$ & $\begin{array}{l}996 \\
82.8\end{array}$ & $\begin{array}{l}101 \\
31.4\end{array}$ & $\begin{array}{l}051 \\
28.4\end{array}$ & $\begin{array}{l}010 \\
51.4\end{array}$ \\
\hline $\begin{array}{c}\pi_{i}=20 \quad \text { Mean } \\
x^{2}(27)\end{array}$ & $\begin{array}{c}006 \\
130.0\end{array}$ & $\begin{array}{c}027 \\
112.3\end{array}$ & $\begin{array}{l}052 \\
94.6\end{array}$ & $\begin{array}{l}950 \\
88.7\end{array}$ & $\begin{array}{l}976 \\
72.3\end{array}$ & $\begin{array}{l}996 \\
73.4\end{array}$ & $\begin{array}{l}101 \\
29.4\end{array}$ & $\begin{array}{l}051 \\
27.3\end{array}$ & $\begin{array}{r}010 \\
45.2\end{array}$ \\
\hline $\begin{array}{c}\mathrm{n}_{\mathrm{i}}=30 \quad \text { Mean } \\
x^{2}(27)\end{array}$ & $\begin{array}{c}008 \\
180.2\end{array}$ & $\begin{array}{c}029 \\
215.6\end{array}$ & $\begin{array}{c}052 \\
168.3\end{array}$ & $\begin{array}{l}950 \\
75.4\end{array}$ & $\begin{array}{l}977 \\
83.7\end{array}$ & $\begin{array}{l}996 \\
75.9\end{array}$ & $\begin{array}{l}102 \\
42.7\end{array}$ & $\begin{array}{l}052 \\
57: 3\end{array}$ & $\begin{array}{l}011 \\
65.2\end{array}$ \\
\hline
\end{tabular}


Table 7 (Continued)

\begin{tabular}{|c|c|c|c|c|c|c|c|c|c|}
\hline & : 005 & 025 & 050 & 950 & 975 & 995 & $a: 10$ & 05 & 01 \\
\hline \multicolumn{10}{|c|}{ Number of Raters } \\
\hline $\mathrm{n}_{\mathrm{r}}=2 \underset{\chi^{2}(27)}{\text { Mean }}$ & $\begin{array}{c}010 \\
292.6\end{array}$ & $\begin{array}{c}036 \\
344.6\end{array}$ & $\begin{array}{c}062 \\
266.5\end{array}$ & $\begin{array}{c}959 \\
278.5\end{array}$ & $\begin{array}{c}982 \\
232.1\end{array}$ & $\begin{array}{c}998 \\
318.1\end{array}$ & $\begin{array}{l}103 \\
48.3\end{array}$ & $\begin{array}{l}054 \\
71.2\end{array}$ & $\begin{array}{l}013 \\
45.2\end{array}$ \\
\hline $\mathrm{n}_{\mathrm{r}}=5 \underset{\chi^{2}(27)}{\text { Mean }}$ & $\begin{array}{l}005 \\
27.1\end{array}$ & $\begin{array}{l}024 \\
20.9\end{array}$ & $\begin{array}{l}048 \\
40.0\end{array}$ & $\begin{array}{l}946 \\
53.8\end{array}$ & $\begin{array}{l}974 \\
19.7\end{array}$ & $\begin{array}{l}995 \\
28.6\end{array}$ & $\begin{array}{l}102 \\
25.8\end{array}$ & $\begin{array}{l}050 \\
12.7\end{array}$ & $\begin{array}{l}010 \\
21.4\end{array}$ \\
\hline $\mathrm{n}_{\mathrm{r}}=8_{\chi^{2}(27)}^{\text {Mean }}$ & $\begin{array}{l}004 \\
35.2\end{array}$ & $\begin{array}{l}022 \\
38.2\end{array}$ & $\begin{array}{l}045 \\
70.4\end{array}$ & $\begin{array}{l}945 \\
67.9\end{array}$ & $\begin{array}{l}973 \\
38.9\end{array}$ & $\begin{array}{l}995 \\
29.6\end{array}$ & $\begin{array}{l}100 \\
29.0\end{array}$ & $\begin{array}{l}049 \\
27.5\end{array}$ & $\begin{array}{l}009 \\
30.4\end{array}$ \\
\hline
\end{tabular}

Note. Decimal points omitted in alphas and proportions.

The standardized estimate is the value

$\left[\left(1-\hat{\rho}^{2}\right)^{1 / 3}-\left(1-\rho^{2}\right)^{1 / 3}\right] /\left[\widehat{\operatorname{Var}}\left(1-\hat{\rho}^{2}\right)^{1 / 3}\right]^{1 / 2}$. 
26 of 27 observed values fell within two standard errors:

$$
2[(.05 \times .95) / 2500]^{1 / 2}=.0087 \text {. }
$$

For nominal alpha of $.01,23$ of 27 observed values fell within two standard errors:

$$
2[(.01 \times .99) / 2500]^{1 / 2}=.0039 \text {. }
$$

For the conditions in which $\rho_{1}^{2}=.7$ (Table 5), 24 of 27 observed values fell within two standard errors of nominal alpha of $.10,23$ of 27 fell within two standard errors of .05 , and 21 of 27 fell within two standard errors of .01 . For coefficient values of $\rho_{1}^{2}=.9$ (Table 6), 25 of 27 observed values fell within two standard errors of nominal alpha level of $10 ; 25$ of 27 fell within two standard errors of nominal .05 alpha, and 24 of 27 fell within two standard errors of nominal .01 alpha.

From inspection of Table 7 it can be seen that the empirical proportion of standardized estimates falling below any given percentile value generally was very close to the corresponding nominal value.

The set of results showing the greatest discrepancy from nominal percentile values is that based on two raters $\left(n_{r}=2\right)$. Although the overall empirical alpha levels tended to be reasonably close to their nominal values, the empirical proportions indicate asymmetry in the results. More of the standardized estimates fell into the lower tail of the normal distribution than would be expected by chance. In addition, the chi-square statistics for the $n_{r}=2$ 
conditions are larger than those for other conditions. This result suggests that there was greater bias in these results than was seen for other values of ${ }_{n}$. The reason for this bias is not clear. It is possible that bias in the standardized estimate is responsible for the asymmetry in the results. Another possibility is that for cases with small numbers of facet conditions the normal distribution is not a good model for the distribution of standardized estimates. It is also possible that the statistic has not reached its asymptotic value in these conditions. To investigate this problem the distribution of the normalized coefficients and their variances would need to be examined.

\section{Design VII with Fixed Item Facet}

The results for the nine conditions in which the Item facet was fixed are presented in Table 8 . These results generally indicate reasonably good maintenance of Type I error control. However, these empirical results do not appear to be quite as close to nominal levels as were those obtained for Design VII with both facets random. The conditions with $n_{r}=2$ deviated most from the nominal proportions.

\section{Design VII with Finite Rater Facet}

The results for the 18 conditions in which Raters was a finite facet are presented in Table 9. Overall, these results indicate good control of Type I error for both 
Table 8

Proportion of the Standardized Estimates Falling below Selected Percentile Points of the unit Normal Distribution and Actual Proportion of Type I Errors (Design VII, Item Facet Fixed)

\begin{tabular}{rllllllllllllll}
\hline$n_{p}$ & $n_{i}$ & $n_{r}$ & $p^{2}$ & $p: 005$ & 025 & 050 & 950 & 975 & 995 & $a: 10$ & 05 & 01 \\
\hline 25 & 20 & 8 & .5 & 008 & 026 & 049 & 941 & 968 & 994 & 108 & 057 & 014 \\
25 & 20 & 8 & .7 & 004 & 024 & 042 & 942 & 969 & 993 & 100 & 055 & 011 \\
25 & 20 & 8 & .9 & 007 & 023 & 052 & 937 & 970 & 992 & 115 & 053 & 014 \\
75 & 30 & 2 & .5 & 008 & 038 & 069 & 962 & 980 & 996 & 106 & 058 & 012 \\
75 & 30 & 2 & .7 & 012 & 042 & 069 & 962 & 979 & 998 & 106 & 063 & 014 \\
75 & 30 & 2 & .9 & 006 & 027 & 049 & 966 & 983 & 998 & 084 & 044 & 008 \\
150 & 10 & 5 & .5 & 006 & 028 & 055 & 942 & 970 & 994 & 113 & 057 & 012 \\
150 & 10 & 5 & .7 & 002 & 017 & 046 & 943 & 970 & 992 & 102 & 047 & 010 \\
150 & 10 & 5 & .9 & 004 & 024 & 045 & 943 & 970 & 992 & 102 & 053 & 012 \\
\hline
\end{tabular}

Note. Decimal points omitted.

The standardized estimate is the value

$\left[\left(1-\hat{\rho}^{2}\right)^{1 / 3}-\left(1-\rho^{2}\right)^{1 / 3}\right] /\left[\widehat{\operatorname{Var}}\left(1-\hat{\rho}^{2}\right)^{1 / 3}\right]^{1 / 2}$. 
Table 9

Proportion of the Standardized Estimates Falling below Selected Percentile Points of the Unit Normal Distribution and Actual Proportion of Type I Errors (Design VII, Rater Facet Finite)

\begin{tabular}{|c|c|c|c|c|c|c|c|c|c|c|c|c|c|}
\hline $\mathrm{n}_{\mathrm{p}}$ & $n_{i}$ & $\mathrm{n}_{I}$ & $\rho^{2}$ & $\mathrm{p}:$ & 005 & 025 & 050 & 950 & 975 & 995 & $a: 10$ & 05 & 01 \\
\hline \multicolumn{14}{|c|}{ Sampling Fraction $=.10$} \\
\hline 25 & 20 & 8 & .5 & & 005 & 024 & 045 & 944 & 970 & 992 & 101 & 054 & 011 \\
\hline 25 & 20 & 8 & .7 & & 005 & 022 & 047 & 944 & 968 & 992 & 102 & 055 & 013 \\
\hline 25 & 20 & 8 & .9 & & 002 & 020 & 042 & 940 & 970 & 993 & 104 & 050 & 009 \\
\hline 75 & 30 & 2 & .5 & & 009 & 033 & 055 & 958 & 981 & 998 & 097 & 052 & 011 \\
\hline 75 & 30 & 2 & .7 & & 007 & 030 & 056 & 950 & 973 & 996 & 106 & 058 & 011 \\
\hline 75 & 30 & 2 & .9 & & 011 & 034 & 063 & 953 & 980 & 999 & 110 & 055 & 012 \\
\hline 150 & 10 & 5 & .5 & & 008 & 030 & 050 & 946 & 974 & 994 & 104 & 057 & 015 \\
\hline 150 & 10 & 5 & .7 & & 005 & 029 & 058 & 951 & 969 & 993 & 107 & 060 & 012 \\
\hline 150 & 10 & 5 & .9 & & 005 & 022 & 041 & 954 & 974 & 996 & 088 & 048 & 009 \\
\hline \multicolumn{14}{|c|}{ Sampling Fraction $=.20$} \\
\hline 25 & 20 & 8 & .5 & & 005 & 024 & 048 & 939 & 967 & 992 & 109 & 058 & 012 \\
\hline 25 & 20 & 8 & .7 & & 006 & 020 & 041 & 940 & 967 & 993 & 101 & 054 & 014 \\
\hline 25 & 20 & 8 & .9 & & 004 & 024 & 044 & 937 & 971 & 995 & 107 & 053 & 009 \\
\hline 75 & 30 & 2 & .5 & & 006 & 027 & 054 & 956 & 980 & 996 & 098 & 048 & 010 \\
\hline 75 & 30 & 2 & .7 & & 008 & 031 & 059 & 948 & 975 & 996 & 111 & 056 & 012 \\
\hline 75 & 30 & 2 & .9 & & 007 & 035 & 059 & 956 & 980 & 994 & 102 & 056 & 013 \\
\hline 150 & 10 & 5 & .5 & & 004 & 022 & 047 & 950 & 971 & 994 & 096 & 052 & 010 \\
\hline 150 & 10 & 5 & .7 & & 004 & 020 & 045 & 938 & 968 & 994 & 107 & 052 & 010 \\
\hline 150 & 10 & 5 & .9 & & 006 & 025 & 056 & 946 & 975 & 996 & 110 & 050 & 010 \\
\hline
\end{tabular}

Note. Decimal points omitted.

The standardized estimate is the value

$\left[\left(1-\hat{\rho}^{2}\right)^{1 / 3}-\left(1-\rho^{2}\right)^{1 / 3}\right] /\left[\widehat{\operatorname{Var}}\left(1-\hat{\rho}^{2}\right)^{1 / 3}\right]^{1 / 2}$. 
sampling fractions used in the study. The size of the sampling fraction does not seem to be influential. Again, in these results, the conditions with $n_{r}=2$ deviated most from the nominal proportions.

\section{Design V-B with Both Facets Random}

Results for the nine conditions of Design $V-B$ in which the Rater facet was nested within persons are presented in Table 10. These results again indicate fairly good control of Type I error. The conditions in which $n_{r}=2$ demonstrated the same sort of asymmetry as was seen in other results.

The Treatment of Negative Variance Components

Negative variance component estimates can arise, because of sampling error, when a variance component is equal to zero in the population. Two ways of dealing with negative variance component estimates were investigated in this thesis. For the first approach no adjustment was made for negative estimates; the CG and the associated variance estimate were calculated from the estimated mean squares. For the second approach negative estimates of variance components were set to zero. The zero components were incorporated in the formulas for other variance component estimates. As a result of this procedure, when a negative estimate was encountered, both the CG and its estimated variance differed from those estimated by the first approach. When no negative components are encountered the 
Table 10

Proportion of the Standardized Estimates Falling below Selected Percentile Points of the Unit Normal Distribution and Actual Proportion of Type I Errors (Design V-B, both Facets Random)

\begin{tabular}{rrrlllllllllll}
\hline$n_{\mathrm{p}}$ & $n_{i}$ & $n_{I}$ & $\rho^{2}$ & $p:$ & 005 & 025 & 050 & 950 & 975 & 995 & $a: 10$ & 05 & 01 \\
\hline 25 & 20 & 8 & .5 & 005 & 022 & 042 & 935 & 972 & 995 & 106 & 050 & 010 \\
25 & 20 & 8 & .7 & 002 & 020 & 043 & 948 & 972 & 993 & 095 & 048 & 010 \\
25 & 20 & 8 & .9 & 006 & 022 & 043 & 930 & 967 & 994 & 113 & 055 & 012 \\
75 & 30 & 2 & .5 & 012 & 036 & 060 & 960 & 982 & 998 & 100 & 054 & 013 \\
75 & 30 & 2 & .7 & 010 & 038 & 066 & 954 & 980 & 999 & 111 & 058 & 011 \\
75 & 30 & 2 & .9 & 006 & 032 & 063 & 951 & 980 & 995 & 112 & 052 & 011 \\
150 & 10 & 5 & .5 & 006 & 026 & 051 & 941 & 969 & 995 & 110 & 058 & 011 \\
150 & 10 & 5 & .7 & 006 & 026 & 051 & 957 & 981 & 998 & 094 & 045 & 008 \\
150 & 10 & 5 & .9 & 006 & 023 & 047 & 951 & 975 & 993 & 096 & 048 & 012 \\
\hline
\end{tabular}

Note. Decimal points omitted.

The standardized estimate is the value

$\left[\left(1-\hat{p}^{2}\right)^{1 / 3}-\left(1-\rho^{2}\right)^{1 / 3}\right] /\left[\widehat{\operatorname{Var}}\left(1-\hat{p}^{2}\right)^{1 / 3}\right]^{1 / 2}$. 
two approaches yield identical estimates.

A total of eight conditions were simulated to compare the two approaches. In the four conditions in which ${ }_{p}=25$ two separate runs of 2,500 replications were conducted for each condition. The runs differed only in the magnitude of their population variance components. Because the results for the two runs did not differ appreciably the results were combined into a single condition based on 5,000 replications. In half of the conditions investigated the $\sigma^{2}$ pi component was set to zero; the $\sigma_{\mathrm{pr}}^{2}$ component was set to zero in the remaining four conditions. In all conditions this resulted in negative component estimates in approximately half of the replications.

The results comparing the two approaches to the treatment of negative variance component estimates are presented in Table 11. The two approaches differed very little in their quality of Type I error control. There was some tendency for the zero substitution approach (Approach 2) to yield fewer rejections in the lower tail of the normal distribution than were given by the first approach. However, the difference is slight and likely is of little practical consequence.

\section{Design VII with Dichotomous Data}

Eight conditions were run in which the item scores were converted to dichotomous data by assigning to scores below 
Table 11

Proportion of the Standardized Estimates Falling below Selected

Percentile Points of the Unit Normal Distribution and Actual

Proportion of Type I Errors for Two Treatments of Negative Variance Component Estimates (Design VII, Both Facets Random)

\begin{tabular}{|c|c|c|c|c|c|c|c|c|c|c|c|c|c|}
\hline${ }^{n_{p}}$ & $n_{i}$ & $\Omega_{r}$ & $\rho^{2}$ & 005 & 025 & 050 & 950 & 975 & 995 & $a:$ & 10 & 05 & 01 \\
\hline \multirow[t]{3}{*}{25} & 10 & 2 & $.5 \quad \sigma_{\mathrm{P} i}^{2}=0$ & & & & & & & & & & \\
\hline & & \multirow{2}{*}{\multicolumn{2}{|c|}{$\begin{array}{l}\text { Approach } 1 \\
\text { Approach } 2\end{array}$}} & 017 & 048 & 077 & 962 & 986 & 999 & & 114 & 062 & 018 \\
\hline & & & & 017 & 048 & 075 & 962 & 985 & 999 & & 113 & 062 & 018 \\
\hline \multirow[t]{3}{*}{25} & 10 & 2 & $.5 \quad \sigma_{\mathrm{pr}}^{2}=0$ & & & & & & & & & & \\
\hline & & \multirow{2}{*}{\multicolumn{2}{|c|}{$\begin{array}{l}\text { Approach } 1 \\
\text { Approach } 2\end{array}$}} & 004 & 021 & 044 & 948 & 975 & 995 & & 096 & 046 & 009 \\
\hline & & & & 003 & 019 & 038 & 942 & 971 & 994 & & 096 & 047 & 009 \\
\hline \multirow[t]{3}{*}{25} & 10 & 2 & $.9 \quad \sigma_{\mathrm{p} i}^{2}=0$ & & & & & & & & & & \\
\hline & & \multicolumn{2}{|c|}{ Approach 1} & 015 & 040 & 071 & 967 & 986 & 999 & & 104 & 054 & 016 \\
\hline & & \multicolumn{2}{|c|}{ Approach 2} & 014 & 038 & 066 & 964 & 985 & 998 & & 103 & 052 & 016 \\
\hline \multirow[t]{3}{*}{25} & 10 & 2 & $.9 \quad \sigma_{\mathrm{pr}}^{2}=0$ & & & & & & & & & & \\
\hline & & \multirow{2}{*}{\multicolumn{2}{|c|}{$\begin{array}{l}\text { Approach } 1 \\
\text { Approach } 2\end{array}$}} & 007 & 028 & 053 & 947 & 977 & 996 & & 108 & 051 & 011 \\
\hline & & & & 004 & 020 & 039 & 937 & 969 & 996 & & 102 & 051 & 009 \\
\hline
\end{tabular}


Table 11 (continued)

\begin{tabular}{|c|c|c|c|c|c|c|c|c|c|c|c|c|c|}
\hline $\mathrm{n}_{\mathrm{p}}$ & $n_{i}$ & $n_{I}$ & $\rho^{2}$ & 005 & 025 & 050 & 950 & 975 & 995 & $a:$ & 10 & 05 & 01 \\
\hline \multirow[t]{2}{*}{75} & 20 & 5 & $.5 \quad \sigma_{\mathrm{p} i}^{2}=0$ & & & & & & & & & & \\
\hline & & & $\begin{array}{l}\text { pproach } 1 \\
\text { pproach } 2\end{array}$ & $\begin{array}{l}006 \\
006\end{array}$ & $\begin{array}{l}024 \\
024\end{array}$ & $\begin{array}{l}052 \\
050\end{array}$ & $\begin{array}{l}944 \\
943\end{array}$ & $\begin{array}{l}975 \\
975\end{array}$ & $\begin{array}{l}994 \\
994\end{array}$ & & $\begin{array}{l}108 \\
107\end{array}$ & $\begin{array}{l}049 \\
049\end{array}$ & $\begin{array}{l}011 \\
012\end{array}$ \\
\hline \multirow[t]{3}{*}{75} & 20 & 5 & $.5 \quad \sigma_{\mathrm{pr}}^{2}=0$ & & & & & & & & & & \\
\hline & & & pproach 1 & 004 & 026 & 043 & 937 & 968 & 991 & & 106 & 058 & 013 \\
\hline & & & pproach 2 & 004 & 024 & 042 & 936 & 967 & 990 & & 106 & 057 & 013 \\
\hline \multirow[t]{3}{*}{75} & 20 & 5 & -9 $\quad \sigma_{\mathrm{pi}}^{2}=0$ & & & & & & & & & & \\
\hline & & & pproach 1 & 008 & 024 & 050 & 951 & 976 & 996 & & 100 & 048 & 012 \\
\hline & & & pproach 2 & 008 & 024 & 050 & 951 & 975 & 996 & & 099 & 049 & 012 \\
\hline \multirow[t]{3}{*}{75} & 20 & 5 & $.9 \quad o_{\mathrm{pr}}^{2}=0$ & & & & & & & & & & \\
\hline & & & pproach 1 & 003 & 028 & 052 & 949 & 974 & 994 & & 102 & 054 & 000 \\
\hline & & & pproach 2 & 000 & 017 & 030 & 930 & 966 & 993 & & 100 & 051 & 007 \\
\hline
\end{tabular}

Note. Approach 1: no adjustment for negative variance component estimates; Approach 2: zero substitution for negative estimates. Conditions where $n_{p}=25$ are based on 5000 replications. Decimal points omitted.

The standardized estimate is the value $\left[\left(1-\hat{\rho}^{2}\right)^{1 / 3}-\left(1-\rho^{2}\right)^{1 / 3}\right] /\left[\widehat{\operatorname{Var}}\left(1-\hat{\rho}^{2}\right)^{1 / 3}\right]^{1 / 2}$. 
the population mean the value zero and to scores at or above the mean the value one. The transformed population coefficient used in constructing the standardized estimate was estimated by simulating the coefficient. The results for conditions using dichotomous data are presented in the upper portion of Table 12. In the lower portion of this table, results for the corresponding conditions using continuous data are presented for purpose of comparison.

In the three conditions where $n_{p}=25$ and $n_{r}=2$ the obtained alpha levels are in excess of the nominal levels. Inspection of the proportions falling below percentile points in each tail indicates marked asymmetry in the results. Many more standardized estimates fell into the lower tail of the normal distribution than fell into the upper tail. When the number of raters was increased to five the results improved somewhat. The empirical alpha levels were closer to nominal alpha although asymmetry was still apparent in these results. The results for $n_{p}=75$ and ${ }_{r}=5$ were similar to those obtained for $n_{p}=25$ and ${ }_{r}=5$. This finding suggests that the size of ${ }_{r}$ plays a more crucial role in determining the quality of Type I error control than does the magnitude of $n_{p}$. This pattern is consistent with that found with continuous data. Generally these results are not as good as those obtained with continuous data. 
Table 12

Proportion of the Standardized Estimates Falling below Selected Percentile Points of the Unit Normal Distribution and Actual Proportion of Type I Errors for Dichotomized Data and Comparable Conditions with Continuous Data (Design VII, both Facets Random)

\begin{tabular}{|c|c|c|c|c|c|c|c|c|c|c|c|c|c|}
\hline $\mathrm{n}_{\mathrm{p}}$ & $\mathrm{n}_{\mathrm{i}}$ & $\mathrm{n}_{\mathrm{r}}$ & $\rho^{2}$ & $\mathrm{p}:$ & 005 & 025 & 050 & 950 & 975 & 995 & $a: 10$ & 05 & 01 \\
\hline \multicolumn{14}{|c|}{ Dichotomized } \\
\hline 25 & 10 & 2 & .4044 & & 020 & 051 & 086 & 968 & 988 & 999 & 118 & 063 & 021 \\
\hline 25 & 10 & 2 & .5995 & & 019 & 048 & 076 & 975 & 994 & 1000 & 101 & 055 & 019 \\
\hline 25 & 10 & 2 & .7956 & & 023 & 060 & 089 & 959 & 982 & 998 & 130 & 07.8 & 026 \\
\hline 25 & 10 & 5 & .4155 & & 010 & 040 & 067 & 955 & 979 & 998 & 112 & 061 & 012 \\
\hline 25 & 10 & 5 & .8431 & & 008 & 027 & 056 & 972 & 985 & 998 & 084 & 042 & 010 \\
\hline 75 & 20 & 5 & .4582 & & 009 & 034 & 063 & 960 & 985 & 998 & 104 & 049 & 012 \\
\hline 75 & 20 & 5 & .6593 & & 004 & 030 & 058 & 961 & 979 & 997 & 098 & 051 & 007 \\
\hline 75 & 20 & 5 & .8656 & & 005 & 028 & 054 & 958 & 986 & 997 & 096 & 042 & 008 \\
\hline \multicolumn{14}{|c|}{ Continuous Data } \\
\hline 2.5 & 10 & 2 & .5 & & 013 & 038 & 060 & 960 & 981 & 997 & 100 & 057 & 016 \\
\hline 25 & 10 & 2 & .7 & & 013 & 045 & 070 & 964 & 986 & 998 & 106 & 058 & 015 \\
\hline 25 & 10 & 2 & .9 & & 016 & 047 & 078 & 969 & 987 & 1000 & 109 & 060 & 016 \\
\hline 25 & 10 & 5 & .5 & & 004 & 022 & 045 & 944 & 974 & 997 & 101 & 049 & 007 \\
\hline 25 & 10 & 5 & .9 & & 004 & 022 & $0 \div 1$ & 945 & 973 & 994 & 096 & 049 & 010 \\
\hline 75 & 20 & 5 & .5 & & 005 & 024 & $0 \div 4$ & 942 & 972 & 996 & 102 & 053 & 009 \\
\hline 75 & 20 & 5 & .7 & & 004 & 025 & 048 & 951 & 974 & 998 & 097 & 052 & 006 \\
\hline 75 & 20 & 5 & .9 & & 004 & 022 & 052 & 943 & 972 & 994 & 108 & 050 & 010 \\
\hline
\end{tabular}

Note. Decimal points omitted.

The standardized estimate is the value

$\left[\left(1-\hat{\rho}^{2}\right)^{1 / 3}-\left(1-\rho^{2}\right)^{1 / 3}\right] /\left[\widehat{\operatorname{Var}}\left(1-\hat{\rho}^{2}\right)^{1 / 3}\right]^{1 / 2}$. 
Chapter 5

\section{Conclusions and a Worked Example of the Procedures}

The final chapter of this thesis has two purposes. First, the implications of the empirical results and directions for future research are presented. The chapter then concludes with an example of how the asymptotic variance expression can be applied to data.

\section{Some Observations about the Empirical Results}

The asymptotic variance expression. The major purpose of this research was to develop asymptotic variance expressions for CGs under a variety of realistic experimental designs. The value of such an expression is twofold. First, the variance expression can be used to construct a confidence interval for the coefficient. Second, the variance can be used to develop procedures for making statistical inferences about sample coefficients.

The results of the empirical simulations performed in this thesis indicate that the variance expressions for the transformed CGs performed well in controlling Type I error in the majority of conditions examined.

The results suggest that the variance expressions can be used to set confidence intervals for designs like those used in the empirical study. Although the results were not as precise for cases with ${ }_{r}=2$ and with dichotomous data as they were for other conditions, the approximate 
confidence intervals in these less optimal conditions still provide a good estimate of the range of the parameter value.

A more cautious approach likely is warranted in using the asymptotic variance expression for hypothesis testing. Results for conditions with dichotomous data and for a facet represented by two conditions indicated unequal rejection rates in the two tails of the normal distribution when hypotheses of the form $H_{0}: \rho^{2}=\rho_{0}^{2}$ are tested with the statistic

$$
k=\left[\left(1-\hat{\rho}^{2}\right)^{1 / 3}-\left(1-\rho_{0}^{2}\right)^{1 / 3}\right] /\left[\widehat{\operatorname{Var}}\left(1-\hat{\rho}^{2}\right)\right]^{1 / 2} .
$$

The test statistic is more likely to reject a true null hypothesis when the estimated $C G$ is larger than the hypothesized population parameter than when the estimated value is smaller than the hypothesized parameter (a large standardized value is associated with a small coefficient value).

To illustrate, consider the case where ${ }_{p}=25$, $n_{i}=30, n_{r}=2$, and $\rho_{1}^{2}=.9$. Testing the hypothesis $H_{0}: \rho_{1}^{2}=.9$ when $\hat{\rho}_{1}^{2}>\rho_{1}^{2}$, the empirical results indicate that the true null hypothesis will be rejected $10.8 \%$ of the time at the nominal $5 \%$ alpha level. In contrast, when testing the hypothesis $\mathrm{H}_{0}: p_{1}^{2}=.9$ when $\hat{p}_{1}^{2}<.9$, the true null hypothesis will be rejected only $2.8 \%$ of the time at the nominal $5 \%$ level. (It should be kept in mind, in connection with the above, that, if we consider this as a 
standard two-tailed test and do not specify the direction of the estimate relative to the hypothesized parameter the actual overall Type I error, at the nominal .05 level, is .068$.

Treatment of negative variance component estimates. A side study was conducted to investigate the effects of two treatments of negative variance component estimates. The two methods resulted in very similar Type I error control. This finding suggests that the technique for deriving the asymptotic variance expression for the transformed coefficient can be applied with either method of dealing with negative variance component estimates. However, practical concerns indicate that negative variance component estimates should be left negative. When a negative estimate is encountered in practice and replaced with a zero value, the expression for estimating the CG changes. Then the expression for the asymptotic variance estimate, derived by application of the delta method, is different from that derived in the absence of a negative variance estimate. To further complicate matters, the form of the new variance expression depends upon which variance component is negative. For the practitioner, it is more straightforward to ignore negative variance component estimates and work only with the mean squares. 
Implications of the Study for the Use of Generalizability Theory

The results of the empirical study investigating the adequacy of the proposed asymptotic variance expression have implications for practical application of the technique. The previously discussed results for the treatment of negative variance component estimates have implications for practice. These results indicate that negative estimates can be left negative without reducing the precision of the technique. Results obtained with small numbers of raters and with dichotomous data also have implications for the application of the techniques developed in this thesis.

Small number of raters. It was seen that the results for conditions with $n_{r}=2$ tended to be asymmetric. The null hypothesis was rejected too often when the sample value of the CG was larger than the true population parameter. Coefficients lower than the true value, however, were not rejected as often as would be expected. One solution to this problem is to plan studies using more than two facet conditions. However, in many circumstances this solution is not a practical one. For example, it may be too expensive to increase the number of raters used in a study beyond two. Similarly, if a study has an Occasion facet, as in a test-retest design, it may not be possible or desirable to have subjects repeat the test or questionnaire more than once. Of course, when it is possible to do so, studies 
should use more than two facet conditions. When this is not practical, the researcher can use the technique and acknowledge the approximate nature of the results. Alternatively, she or he can adjust the critical value for the significance test according to whether the observed result is lower or higher than the hypothesized parameter value. For example, if the observed CG is larger than the hypothesized value, the researcher could test the null hypothesis at the .035 level of significance instead of at the .05 level. Similarly, if the observed $C G$ is smaller than the hypothesized value, a less stringent alpha level can be used to test the null hypothesis.

\section{Dichotomous data. The results for conditions with} dichotomous data also indicated that results falling into the rejection region were not evenly divided between the two tails of the normal distribution. Further, with two raters, the Type I error control was not maintained. To overcome these shortcomings, the researcher, again, could adjust critical values according to whether the sample CG is higher or lower than the hypothesized value. For example, where $n_{p}=25, n_{i}=10, n_{r}=2$, and $\rho_{1}^{2}=.7956$, the null hypothesis would be rejected approximately $12 \%$ of the time at the nominal $5 \%$ alpha level when the sample $C G$ is larger than the population value. When the sample value is smaller than the CG the true nuIl hypothesis is rejected only $3.6 \%$ of the time. Again, if possible, researchers should try to avoid using small numbers of facet conditions. However, this 
approach to the problem is not always feasible and because of the prevalence of dichotomous items in psychological research, the researcher can deal with the asymmetry by adjusting levels of significance according to the magnitude of the sample coefficient. With a sample coefficient larger than the hypothesized population parameter, a $5 \%$ alpha level test should be conducted at a more stringent level of significance. In the case of a coefficient smaller than the hypothesized parameter, a less stringent than nominal level of significance would be used.

\section{Limitations of the Present Study}

As in any empirical simulation study, there are a number of limitations to this investigation imposed by the conditions studied. One such limitation is that it is uncertain what minimum number of facet conditions is required to produce really good Type I error control. Two facet conditions tended to produce results that were asymmetric while the use of five conditions produced good error control in both tails of the distribution. However, it is not known whether three or four conditions would also yield acceptable results. It seems relatively certain that three facet conditions would result in better Type I error control than that realized with two conditions.

Another Iimitation of the present study is that it focused on a small number of ANOVA designs. Numerous, more complicated, ANOVA designs are possible within a measurement 
context. Although the results of this study indicated that the proposed procedures perform well with two facet designs, it is uncertain whether the technique would perform well with designs having larger numbers of facets. It is possible, for example, that the number of estimated mean squares needed for a three facet design would produce large amounts of unsystematic error that would cause the statistic to behave erratically.

One further restriction on the application of the present research results is that the data used in these simulations were sampled from populations with underlying normal distributions. Although the analysis of variance is robust with respect to violation of the normality assumption (see e.g., Glass, Peckham, \& Sanders, 1972), it is not certain that the same claim can be made about the performance of the estimated CG and its associated variance expression. Further research is necessary to determine the effect of the violation of the normality assumption on the performance of these estimates.

\section{Suggestions for Future Research}

The above-mentioned limitations of the present study should be addressed in future research. One such study should investigate the adequacy of the asymptotic variance expression with three and four facet conditions. Research is also needed to investigate the suitability of the procedure to more complicated ANOVA models. Studies should be 
undertaken in which three and four facets (both nested and crossed designs) are used. Future research should also investigate the effects of violating the ANOVA assumption of the data having an underlying normal distribution. Experimental data sometimes do not meet this requirement. It would be desirable to know how the technique performs when the normality assumption is not met. Simulations with data drawn from such distributions as the uniform, lognormal, and exponential would help determine the importance of the normality assumption for application of the technique.

The present research was concerned with testing a null hypothesis about the value of a single population CG. The technique can be extended to develop tests for two or more independent sample coefficients. For two coefficients, a test analogous to the two independent group t-test could be used; for multiple coefficients the technique described by Marascuilo (1966) could be employed. The adequacy of these extensions requires further investigation.

Another extension of this research would be the development of a covariance expression for two dependent sample coefficients. This expression could then be used to test hypotheses about coefficients based on data obtained from dependent samples. For two dependent coefficients a technique analogous to the dependent sample t-test could be used. Multiple dependent coefficients could be tested with a quadratic form chi-square statistic. Again, such statistics 
would require empirical verification before their use could be recommended.

As a final suggestion for future reseach, a study could be undertaken to investigate power of the procedures. The present research was concerned with control of Type I error under a variety of situations. Investigations of the power of the technique would give researchers an indication of the sample sizes and condition numbers necessary for adequate power when using the technique inferentially.

\section{A Worked Example using the Present Procedure}

The application of one of the asymptotic variance expressions developed in this thesis is illustrated in this section using data from the Schroeder et al. (1983) study. The random effects ANOVA results were presented in Table 2. By substituting the observed mean squares into the formulas for the CG and the asymptotic variance expressions (eq. 4 and 5 ), the following values are obtained:

$$
\begin{aligned}
& \hat{\rho}_{1}^{2}=.860, \\
& \left(1-\hat{\rho}_{1}^{2}\right)^{1 / 3}=.519, \\
& \widehat{\operatorname{Var}}\left(1-\hat{\rho}_{1}^{2}\right)^{1 / 3}=.00197 .
\end{aligned}
$$

The $9.5 \%$ confidence interval is constructed as

$$
\operatorname{Pr}\left\{\left[\left(1-\hat{\rho}_{1}^{2}\right)^{1 / 3}-1.96 \hat{o}^{*}\right]<\left(1-\rho_{1}^{2}\right)^{1 / 3}\right.
$$




$$
\left.<\left[\left(1-\hat{\rho}_{1}^{2}\right)^{1 / 3}+1.96 \hat{\sigma}^{*}\right]\right\} \doteq .95
$$

where $\hat{\sigma}^{*}=\left[\widehat{\operatorname{Var}}\left(1-\hat{\rho}_{1}^{2}\right)^{1 / 3}\right]^{1 / 2}$.

After algebraic manipulation, this expression can be written as

$$
\begin{aligned}
& \operatorname{Pr}\left\{1-\left[\left(1-\hat{\rho}_{1}^{2}\right)^{1 / 3}+1.96 \hat{\sigma}\right]^{3}<\rho^{2}\right. \\
& \left.<1-\left[\left(1-\hat{\rho}_{1}^{2}\right)^{1 / 3}-1.96 \hat{\sigma}^{*}\right]^{3}\right\} \doteq .95 .
\end{aligned}
$$

Substitution of the observed values into this equation gives the interval

$$
\operatorname{Pr}\left\{.777<\rho_{1}^{2}<.919\right\} \doteq .95 \text {. }
$$

In addition, a simple null hypothesis of the form $H_{0}$ : $\rho_{1}^{2}=\rho_{0}^{2}$ (where $\rho_{0}^{2}$ is some hypothesized value) can be tested with the statistic. Testing the null hypothesis $H_{0}: \rho_{1}^{2}=.90$ yields a value of

$$
\begin{aligned}
k & =\left[(1-.86)^{1 / 3}-(1-.90)^{1 / 3}\right] /(.00197)^{1 / 2} \\
& =1.235,
\end{aligned}
$$

which is not significant at the .10 level of significance (two-tailed test). Note here that this is a conservative test; $n_{r}=2$ and the estimated CG is smaller than the hypothesized value. 


\section{$\underline{\text { References }}$}

Brennan, R.L., \& Kane, M.T. (1979). Generalizability theory: A review of basic concepts, issues, and procedures. In R.E. Traub (ed.), New directions in testing and measurement. San Francisco: Jossey-Bass.

Cain, P.S., \& Green, B.F. (1983). Reliabilities of selected ratings available from the Dictionary of Occupational Titles. Journal of Applied Psychology, 68, 155-165. Cardinet, J., Tourneur, Y., \& Allal, L. (1976). The symmetry of generalizability theory: Applications to educational measurement. Journal of Educational Measurement, 13, $119-135$.

Cardinet, J., Tourneur, Y., \& Allal, L. (1981). Extension of generalizability theory and its applications in educational measurement. Journal of Educational Measurement, 18, 183-204.

Chalmers, D.J., \& Knight, R.G. (1985). The reliability of ratings of the familiarity of environmental stimuli: A generalizability analysis. Environment and Behavior, 17, $223-238$.

Cronbach, L.J. (1947). Test "reliability": Its meaning and determination. Psychometrika, 12, 1-16.

Cronbach, L.J. (1970). Essentials of psychological testing (3rd ed.). New York: Harper \& Row. 
Cronbach, L.J., Gleser, G.C., Nanda, H., \& Rajaratnam, N. (1972). The dependability of behavioral measurements.

New York: Wiley.

Cronbach, L.J., Rajaratnam, N., \& Gleser, G.C. (1963).

Theory of generalizability: A liberalization of

reliability theory. The British Journal of Statistical

Psychology, 16, 138-163.

Doverspike, D., \& Barrett, G.V. (1984). An internal bias

analysis of a job evaluation instrument. Journal of

Applied Psychology, 69, 648-662.

Doverspike, D., Carlisi, A.M., Barrett, G.V., \& Alexander,

R.A. (1983). Generalizability analysis of a point-method job evaluation instrument. Journal of Applied

Psychology, 68, 476-483.

Farrell, A.D., Mariotto, M.J., Conger, A.J., Curran, J.P., \&

Wallander, J.L. (1979). Self-ratings and judges' ratings

of heterosexual social anxiety and skill: A

generalizability study. Journal of Consulting and

Clinical Psychology, 47, 164-175.

Feldt, I.S. (1965). The approximate sampling distribution of

Kuder-Richardson reliability coefficient twenty.

Psychometrika, 30, 357-370.

Feldt, L.S. (1969). A test of the hypothesis that Cronbach's alpha or kuder-Richardson coefficient twenty is the same for two tests. Psychometrika, 34, 363-373. 
Fleiss, J.L. (1971). On the distribution of a linear combination of independent $\mathrm{Chi}$ squares. Journal of the American Statistical Association, 66, 142-144.

Fleiss, J.L., \& Shrout, P.E. (1978). Approximate interval

estimation for a certain intraclass correlation coefficient. Psychometrika, 43, 259-262.

Fraser, S.L., Cronshaw, S.F., \& Alexander, R.A. (1984).

Generalizability analysis of a point method job evaluation instrument: A field study. Journal of Applied Psychology, 69, 643-647.

Gillmore, G.M., Kane, M.T., \& Naccarato, R.W. (1978). The generalizability of student ratings of instruction. Journal of Educational Measurement, 15, 1-15. Glass, G.V., Peckham, P.D., \& Sanders, J.R. (1972). Consequences of failure to meet assumptions underlying the fixed effects analyses of variance and covariance. Journal of Educational Research, 42, 237-288. Gleser, G.C., Cronbach, L.J., \& Rajaratnam, N. (1965). Generalizability of scores influenced by multiple sources of variance. Psychometrika, 30, 395-418. Gulliksen, H. (1936). The content reliability of a test. Psychometrika, 1, 189-194. Haggard, E.A. (1958). Intraclass correlation and the analysis of variance. New York: Dryden. 
Hakstian, A.R., \& Lind, J.C. (1982, November). Inferential procedures for alpha coefficients. Paper presented at the meeting of the Society of Multivariate Experimental Psychology, Atlanta.

Hakstian, A.R., \& Whalen, T.E. (1976). A k-sample significance test for independent alpha coefficients. Psychometrika, 41, 219-231. Hansen, D.J., Tiscdelle, D.A., \& O'Dell, S.L. (1985). Audio recorded and directly observed parent-child interactions: A comparison of observation methods. Behavioral Assessment, $\underline{7}, 389-399$.

Jackson, D.N., \& Paunonen, S.V. (1980). Personality structure and assessment. In M.R. Rosenzweig \& L.W. Porter (Eds.) Annual Review of Psychology (Vol. 31 ). Palo Alto: Annual Reviews Inc. Kane, M.T., \& Brennan, R.L. (1977). The generalizability of class means. Review of Educational Research, 47, 267-292.

Kane, M.T., Gillmore, G.M., \& Crooks, T.J. (1976). Student evaluations of teaching: The generalizability of class means. Journal of Educational Measurement, 13, 171-183. Knuth, D.E. (1968). The art of computer programming (Vol. 2): Seminumerical algorithms. Reading, MA: Addison-Wesley. 
Lahey, M.A., Downey, R.G., \& Saal, F.E. (1983). Intraclass correlations: There's more than meets the eye. Psychological Bulletin, 93, 586-595.

Leone, F.C., \& Nelson, L.S. (1966). Sampling distributions of variance components I. Empirical studies of balanced nested designs. Technometrics, $8,457-468$.

Marascuilo, L.A. (1966). Large sample multiple comparisons. Psychological Bulletin, 65, 280-290.

Millman, J., \& Glass, G.V (1967). Rules of thumb for writing the ANOVA table. Journal of Educational Measurement, 4 , $41-51$.

Mitchell, S.K. (1979). Interobserver agreement, reliability, and generalizability of data collected in observational studies. Psychological Bulletin, 86, 376-390.

Nunnally, J.C. (1978). Psychometric Theory (2nd ed.). New York: McGraw-Hill.

Nussbaum, A. (1984). Multivariate generalizability theory in educational measurement: An empirical study. Applied Psychological Measurement, $8,219-230$.

Paulson, E. (1942). An approximate normalization of the analysis of variance distribution. Annals of Mathematical Statistics, 13, 233-235. Rajaratnam, N., Cronbach, L.J., \& Gleser, G.C. (1965). Generalizability of stratified-parallel tests. Psychometrika, 30, 39-56. 
Rao, C.R. Linear statistical inference and its applications (2nd ed.). New York: Wiley.

Rentz, R.R. (1980). Rules of thumb for estimating

reliability coefficients using generalizability theory.

Educational and Psychological Measurement, 40, 575-592.

Satterthwaite, F.E. (1941). Synthesis of variance.

Psychometrika, 6, 309-316.

Satterthwaite, F.E. (1946). An approximate distribution of

estimates of variance components. Biometrics Bulletin, $\underline{2}, 110-114$.

Scheffê (1959). The analysis of variance (2nd ed.). New

York: Wiley.

Schroeder, M.L., Schroeder, K.G., \& Hare, R.D. (1983).

Generalizability of a checklist for assessment of

psychopathy. Journal of Consulting and Clinical

Psychology, 51, 511-516.

Shavelson, R.J., \& Webb, N.M. (1981). Generalizability

theory: 1973-1980. British Journal of Mathematical and

Statistical Psychology, 34, 133-166.

Shrout, P.E., \& Eleiss, J.L. (1979). Intraclass

correlations: Uses in assessing rater reliability.

Psychological Bulletin, 86, 420-428.

Smith, P.L. (1978). Sampling errors of variance components

in small sample multifacet generalizability studies. Journal of Educational Statistics, $3,319-346$. 
Wallender, J.L., Conger, A.J., \& Conger, J.C. (1985). Development and evaluation of a behaviorally referenced rating system for heterosocial skills. Behavioral Assessment, I, 137-153.

Wiggins, J.S. (1973). Personality and prediction: Principles of personality assessment. Reading, MA: Addison-Wesley. Wilson, E.B., \& Hilferty, M.M. (1931). The distribution of chi-square. Proceedings of the National Academy of Science, $17,684-688$. Winer, B.J. (1971). Statistical principles in experimental design (2nd ed.). New York: McGraw-Hill. 
Appendix A

Values of the Population Variance Components 
Values of Population Variance Components

1. Design VII with Item Facet Fixed

\begin{tabular}{lrrrrrrr}
\hline$\rho^{2}$ & $n_{p}$ & $n_{i}$ & $n_{r}$ & $\sigma_{\mathrm{p}}^{2}$ & $\sigma_{\mathrm{pi}}^{2}$ & $\sigma_{\mathrm{pr}}^{2}$ & $\sigma_{\mathrm{pi}, \boldsymbol{c}}^{2} \epsilon$ \\
\hline .5 & 25 & 20 & 8 & .303 & .250 & 3.240 & 3.000 \\
.7 & 25 & 20 & 8 & .434 & .250 & 1.275 & 3.000 \\
.9 & 25 & 20 & 8 & .566 & .250 & .225 & 3.000 \\
.5 & 75 & 30 & 2 & .733 & 1.500 & 1.467 & 3.000 \\
.7 & 75 & 30 & 2 & .903 & 1.500 & .660 & 3.000 \\
.9 & 75 & 30 & 2 & 1.790 & 1.500 & .220 & 3.000 \\
.5 & 150 & 10 & 5 & .330 & 1.000 & 2.250 & 2.000 \\
.7 & 150 & 10 & 5 & .542 & 1.000 & 1.190 & 2.000 \\
.9 & 150 & 10 & 5 & .754 & 1.000 & .130 & 2.000 \\
\hline
\end{tabular}

2. Design VII with Rater Facet Finite

\begin{tabular}{|c|c|c|c|c|c|c|c|}
\hline$\rho^{2}$ & ${ }^{n} p$ & $n_{i}$ & $n_{r}$ & $\sigma_{\mathrm{p}}^{2}$ & $\sigma_{\mathrm{pi}}^{2}$ & \multicolumn{2}{|c|}{$\sigma_{\text {pir, } \epsilon}^{2}$} \\
\hline \multicolumn{8}{|c|}{ Sampling Fraction $=.10$} \\
\hline $\begin{array}{l}.5 \\
.7 \\
.9 \\
.5 \\
.7 \\
.9 \\
.5 \\
.7 \\
.9\end{array}$ & $\begin{array}{r}25 \\
25 \\
25 \\
75 \\
75 \\
75 \\
150 \\
150 \\
150\end{array}$ & $\begin{array}{l}20 \\
20 \\
20 \\
30 \\
30 \\
30 \\
10 \\
10 \\
10\end{array}$ & $\begin{array}{l}8 \\
8 \\
8 \\
2 \\
2 \\
2 \\
5 \\
5 \\
5\end{array}$ & $\begin{array}{l}.964 \\
.997 \\
.984 \\
.955 \\
.980 \\
.990 \\
.968 \\
.986 \\
.989\end{array}$ & $\begin{array}{r}6.340 \\
2.684 \\
.669 \\
3.860 \\
1.096 \\
.315 \\
3.724 \\
1.538 \\
.301\end{array}$ & $\begin{array}{r}5.735 \\
2.435 \\
.585 \\
1.823 \\
.790 \\
.190 \\
3.270 \\
1.370 \\
.370\end{array}$ & $\begin{array}{l}.300 \\
.300 \\
.300 \\
.300 \\
.300 \\
.300 \\
.300 \\
.300 \\
.300\end{array}$ \\
\hline \multicolumn{8}{|c|}{ Sampling Fraction $=.20$} \\
\hline $\begin{array}{l}.5 \\
.7 \\
.9 \\
.5 \\
.7 \\
.9 \\
.5 \\
.7 \\
.9\end{array}$ & $\begin{array}{r}25 \\
25 \\
25 \\
75 \\
75 \\
75 \\
150 \\
150 \\
150\end{array}$ & $\begin{array}{l}20 \\
20 \\
20 \\
30 \\
30 \\
30 \\
10 \\
10 \\
10\end{array}$ & $\begin{array}{l}8 \\
8 \\
8 \\
2 \\
2 \\
2 \\
5 \\
5 \\
5\end{array}$ & $\begin{array}{r}.925 \\
.976 \\
1.011 \\
.908 \\
.980 \\
1.004 \\
.935 \\
.978 \\
1.008\end{array}$ & $\begin{array}{r}7.093 \\
2.785 \\
.773 \\
4.770 \\
1.620 \\
.380 \\
4.048 \\
1.616 \\
.324\end{array}$ & $\begin{array}{r}5.685 \\
2.435 \\
.585 \\
1.857 \\
.790 \\
.190 \\
3.270 \\
1.370 \\
.370\end{array}$ & $\begin{array}{l}.300 \\
.300 \\
.300 \\
.300 \\
.300 \\
.300 \\
.300 \\
.300 \\
.300\end{array}$ \\
\hline
\end{tabular}


3. Design $V-B$ with both Facets Random

\begin{tabular}{lllllllll}
\hline$\rho^{2}$ & $n_{p}$ & $n_{i}$ & $n_{r}$ & $\sigma_{\mathrm{p}}^{2}$ & $\sigma_{\mathrm{p} i}^{2}$ & $\sigma_{\mathrm{r}: \mathrm{p}}^{2}$ & $\sigma_{\mathrm{I} r: \mathrm{p}, \epsilon}^{2}$ \\
\hline .5 & 25 & 20 & 8 & .700 & .600 & 5.345 & .300 \\
.7 & 25 & 20 & 8 & .700 & .600 & 2.145 & .300 \\
.9 & 25 & 20 & 8 & .700 & .600 & .367 & .300 \\
.5 & 75 & 30 & 2 & .700 & .600 & 1.350 & .300 \\
.7 & 75 & 30 & 2 & .700 & .600 & .550 & .300 \\
.9 & 75 & 30 & 2 & .700 & .600 & .106 & .300 \\
.5 & 150 & 10 & 5 & .800 & .600 & 3.670 & .300 \\
.7 & 150 & 10 & 5 & .800 & .600 & 1.384 & .300 \\
.9 & 150 & 10 & 5 & .800 & .600 & .114 & .300 \\
\hline
\end{tabular}

4. Design VII with a Zero Variance Component

\begin{tabular}{llllllll}
\hline$\rho^{2}$ & $n_{p}$ & $n_{i}$ & $n_{r}$ & $\sigma_{\mathrm{p}}^{2}$ & $\sigma_{\mathrm{p} i}^{2}$ & $\sigma_{\mathrm{pr}}^{2}$ & $\sigma_{\mathrm{p} i \mathrm{r}, \epsilon}^{2}$ \\
\hline .5 & 25 & 10 & 2 & 1.000 & .000 & 1.970 & .300 \\
.5 & 25 & 10 & 2 & 1.000 & .000 & 1.900 & 1.000 \\
.5 & 25 & 10 & 2 & 1.000 & 9.500 & .000 & 1.000 \\
.5 & 25 & 10 & 2 & 1.000 & 8.000 & .000 & 4.000 \\
.9 & 25 & 10 & 2 & 1.000 & .000 & .122 & 1.000 \\
.9 & 25 & 10 & 2 & 1.000 & .000 & .192 & .300 \\
.9 & 25 & 10 & 2 & 1.000 & .961 & .000 & .300 \\
.9 & 25 & 10 & 2 & 1.000 & .611 & .000 & 1.000 \\
.5 & 75 & 20 & 5 & .500 & .000 & 2.500 & 5.000 \\
.5 & 75 & 20 & 5 & .500 & 9.000 & .000 & 5.000 \\
.9 & 75 & 20 & 5 & 1.000 & .000 & .541 & .300 \\
.9 & 75 & 20 & 5 & 1.000 & .022 & .000 & 10.000 \\
\hline
\end{tabular}

